\title{
Affine and Finite Lie Algebras and Integrable Toda Field Equations on Discrete Space-Time ${ }^{\star}$
}

\author{
Rustem GARIFULLIN ${ }^{\dagger}$, Ismagil HABIBULLIN ${ }^{\dagger}$ and Marina YANGUBAEVA $\ddagger$ \\ $\dagger$ Ufa Institute of Mathematics, Russian Academy of Science, \\ 112 Chernyshevskii Str., Ufa, 450077, Russia \\ E-mail: grustem@gmail.com, habibullinismagil@gmail.com \\ $\ddagger$ Faculty of Physics and Mathematics, Birsk State Social Pedagogical Academy, \\ 10 Internationalnaya Str., Birsk, 452452, Russia \\ E-mail: marina.yangubaeva@mail.ru
}

Received April 24, 2012, in final form September 14, 2012; Published online September 18, 2012 http://dx.doi.org/10.3842/SIGMA.2012.062

\begin{abstract}
Difference-difference systems are suggested corresponding to the Cartan matrices of any simple or affine Lie algebra. In the cases of the algebras $A_{N}, B_{N}, C_{N}, G_{2}, D_{3}$, $A_{1}^{(1)}, A_{2}^{(2)}, D_{N}^{(2)}$ these systems are proved to be integrable. For the systems corresponding to the algebras $A_{2}, A_{1}^{(1)}, A_{2}^{(2)}$ generalized symmetries are found. For the systems $A_{2}, B_{2}$, $C_{2}, G_{2}, D_{3}$ complete sets of independent integrals are found. The Lax representation for the difference-difference systems corresponding to $A_{N}, B_{N}, C_{N}, A_{1}^{(1)}, D_{N}^{(2)}$ are presented.

Key words: affine Lie algebra; difference-difference systems; $S$-integrability; Darboux integrability; Toda field theory; integral; symmetry; Lax pair
\end{abstract}

2010 Mathematics Subject Classification: 35Q53; 37K40

\section{Introduction}

Systems of partial differential equations of the form

$$
r_{x, y}^{i}=e^{\sum_{j=1}^{j=N} a_{i j} r^{j}}, \quad i=1,2, \ldots, N
$$

called generalized two-dimensional Toda lattices have very important applications in Liouville and conformal field theories, they are studied in details (see [3, 5, 6, 9, 10, 11, 13, 27, 28, 29, $30,34,36]$ and the references therein). Here the matrix $A=\left\{a_{i, j}\right\}$ is the Cartan matrix of an arbitrary finite or affine Lie algebra. It is known that in the former case system (1.1) is Darboux integrable while in the latter case $-S$-integrable. The widely known Drinfel'd-Sokolov formalism allows one to construct the Lax representation for the system (1.1) in terms of the Lie algebra canonically associated with the corresponding Cartan matrix $A$.

The problem of finding discrete versions of the system is intensively studied (see, for instance, $[2,14,15,16,17,19,20,21,22,24,25,38,39,40])$. Recently, in [18] integrable differentialdifference analog of system (1.1) was suggested

$$
v_{1, x}^{i}-v_{x}^{i}=e^{\sum_{j=1}^{j=i-1} a_{i, j} v^{j}+\sum_{j=i+1}^{j=N} a_{i, j} v_{1}^{j}+\frac{1}{2} a_{i, i}\left(v^{i}+v_{1}^{i}\right)}, \quad i=1,2, \ldots, N .
$$

Here the functions $v^{j}=v^{j}(n, x), j=1, \ldots, N$ are the searched field variables. The subindex denotes a shift of the discrete variable $n$ or the derivative with respect to $x: r_{k}^{j}=r^{j}(n+k, x)$

${ }^{\star}$ This paper is a contribution to the Special Issue "Geometrical Methods in Mathematical Physics". The full collection is available at http://www.emis.de/journals/SIGMA/GMMP2012.html 
and $r_{x}^{j}=\frac{\partial}{\partial x} r^{j}(n, x)$. A particular case of (1.2), corresponding to the algebra $C_{N}$ is also studied in $[37]$.

In the present article we study the problem of further discretization of system (1.1), i.e. the problem of finding a rule allowing to assign to any Cartan matrix a system of integrable difference-difference equations approximating in the continuum limit system (1.2) and therefore system (1.1). As it was pointed out in [40] the problem of discretization is important from physical viewpoint, they might have applications in discrete field theory and in quantum physics (see also $[22,25,38]$ ). They can also be regarded as difference schemes in numerical computations (see [23]).

Following questions were addressed in [40]:

1) whether there exists an integrable discrete version for any two dimensional Toda field equation (1.1);

2) which kind of algebraic structure (like Lie algebra or Lie group) is naturally related to discrete versions.

The subject has intensively been studied during the last 10-15 years. Various discrete versions of Toda field equations were investigated in literature (see [15, 22, 24, 25, 39] and references therein). As an alternative answer to the first question we suggest a fully discrete version of system (1.1) in the following form

$$
e^{-u_{1,1}^{i}+u_{1,0}^{i}+u_{0,1}^{i}-u_{0,0}^{i}}-1=e^{\sum_{j=1}^{j=i-1} a_{i, j} u_{0,1}^{j}+\sum_{j=i+1}^{j=N} a_{i, j} u_{1,0}^{j}+\frac{1}{2} a_{i, i}\left(u_{0,1}^{i}+u_{1,0}^{i}\right)}, \quad i=1,2, \ldots, N,
$$

which evidently approximates (1.1) and (1.2). Here $u^{j}=u^{j}(n, m), j=1,2, \ldots, N$, is a set of the field variables. The subindex indicates shifts of the $\operatorname{arguments} n, m$ as follows $u_{i, k}^{j}:=$ $u^{j}(n+i, m+k)$. The system corresponding to the algebra $A_{N}$ coincides with that found years ego by Hirota (see [21]). Obviously, system (1.3) is invariant under the replacement $n \leftrightarrow-m$. This property is inherited from the fact that system (1.1) is invariant under the change $x \leftrightarrow y$.

The main result of the present article is in formulating of the conjecture below and proving it for numerous examples of Lie algebras.

\section{Conjecture.}

a) If $A$ is the Cartan matrix of a semi-simple Lie algebra then (1.3) is Darboux integrable, in other words, it admits a complete set of integrals in both directions. Roughly speaking Darboux integrable difference-difference equations can be reduced to ordinary difference equations.

b) If $A$ is the Cartan matrix of an affine Lie algebra then (1.3) is $S$-integrable, i.e. it can be integrated by means of the inverse scattering transform method. It is generally accepted that existence of generalized symmetries or Lax pairs indicates $S$-integrability.

Part $a$ ) of the conjecture is proved for the cases: $A_{2}, B_{2}, C_{2}, G_{2}, D_{3}$ by finding complete sets of integrals. Systems corresponding to the algebras $A_{N}, B_{N}, C_{N}$ are studied by using Lax pair in Section 6. In the cases $A_{2}, B_{2}\left(C_{2}\right), G_{2}$ the exponential system (1.3) reads as follows

$$
e^{-u_{1,1}^{1}+u_{1,0}^{1}+u_{0,1}^{1}-u_{0,0}^{1}}-1=e^{u_{0,1}^{1}+u_{1,0}^{1}-u_{1,0}^{2}}, \quad e^{-u_{1,1}^{2}+u_{1,0}^{2}+u_{0,1}^{2}-u_{0,0}^{2}}-1=e^{-c u_{0,1}^{1}+u_{0,1}^{2}+u_{1,0}^{2}},
$$

where $c$ takes only three values $c=1,2,3$. Here the values $c=1, c=2, c=3$ correspond to the algebras $A_{2}, B_{2}$ or $C_{2}, G_{2}$ respectively. Let us introduce a notation for the nonlinear second-order partial difference operator by setting

$$
\Delta(u):=e^{-u_{1,1}+u_{1,0}+u_{0,1}-u_{0,0}}-1,
$$


then the last system takes a compact form

$$
\Delta\left(u^{1}\right)=e^{u_{0,1}^{1}+u_{1,0}^{1}-u_{1,0}^{2}}, \quad \Delta\left(u^{2}\right)=e^{-c u_{0,1}^{1}+u_{0,1}^{2}+u_{1,0}^{2}} .
$$

Integrals in both directions for system (1.4) are given in Section 2.

Since the map defined in (1.3) converts any $N \times N$ matrix to a system of difference-difference equations one can easily specify the form of systems corresponding to any Cartan matrix canonically related to a finite or affine Lie algebra both of classical series or exceptional. For instance, for the algebra $D_{3}$ with the Cartan matrix

$$
A=\left(\begin{array}{ccc}
2 & -1 & -1 \\
-1 & 2 & 0 \\
-1 & 0 & 2
\end{array}\right)
$$

we have an integrable system of the form

$$
\Delta\left(u^{1}\right)=e^{u_{0,1}^{1}+u_{1,0}^{1}-u_{1,0}^{2}-u_{1,0}^{3}}, \quad \Delta\left(u^{2}\right)=e^{-u_{0,1}^{1}+u_{0,1}^{2}+u_{1,0}^{2}}, \quad \Delta\left(u^{3}\right)=e^{-u_{0,1}^{1}+u_{0,1}^{3}+u_{1,0}^{3}} .
$$

Complete sets of integrals for this system is given in Section 2.

For the Kac-Moody algebra $A_{2}^{(2)}$ with the Cartan matrix

$$
A=\left(\begin{array}{cc}
2 & -1 \\
-4 & 2
\end{array}\right)
$$

the corresponding system is

$$
\Delta\left(u^{1}\right)=e^{u_{0,1}^{1}+u_{1,0}^{1}-u_{1,0}^{2}}, \quad \Delta\left(u^{2}\right)=e^{-4 u_{0,1}^{1}+u_{0,1}^{2}+u_{1,0}^{2}} .
$$

For the difference-difference systems corresponding to the algebras $A_{2}, A_{1}^{(1)}, A_{2}^{(2)}$ generalized symmetries are found, for $A_{2}$ the symmetries have usual form, for $A_{1}^{(1)}, A_{2}^{(2)}$ they have nonlocal form (or, they are of hyperbolic type, see Definition 1.5 below). In the literature generalized symmetries are regarded as a criterion of integrability. Thus one concludes that systems (1.5), (3.1) provide new examples of $2 \times 2 S$-integrable quad graph models.

Stress that the number of the field variables in all three systems (1.1), (1.2), (1.3) coincides with the rank of the corresponding Lie algebra.

It is known that any system of difference-difference equations of hyperbolic type admits a pair of characteristic Lie algebras which are effectively evaluated (see [14]). Characteristic Lie algebras of system (1.3) are closely connected with the Lie algebra canonically related to the given Cartan matrix $A$. In our opinion this relation could allow one to answer the second question in [40] (see the list of questions above).

The problem of developing discrete versions of the Drinfel'd-Sokolov formalism is also challenging since today systems of discrete equations are very popular. They have a large variety of application in theoretical physics, in discrete geometry, in the theory of tau functions in lattice Toda field equations. The present article would also provide an "experimental" background for creating a discrete theory parallel to the Drinfel'd-Sokolov formalism.

The article is organized as follows. In Section 1.1 notions of the integrals and generalized symmetries of both evolutionary and hyperbolic type for quad graph equations are defined. Sufficient condition of complete set of integrals is proved. In Section 2 a method of discretization of Darboux integrable models preserving integrability is discussed. The method is explained in details by an example of the Liouville equation [19]. Then it is applied to the system (1.2) to get its difference-difference analog. The case $B_{2}$ is studied in more details. These simulations allowed us to guess the formula (1.3). Complete sets of independent integrals for the systems $A_{2}, B_{2}, C_{2}, G_{2}, D_{3}$ are presented. 
Generalized symmetries are evaluated for the systems corresponding $A_{2}, A_{1}^{(1)}, A_{2}^{(2)}$ in Section 3. Special attention is paid to hyperbolic type symmetries which provide a semi-discrete version of the consistency around a cube property of quad graph equations [1]. In Section 4 the concept of the characteristic Lie algebra (see [14]) of discrete models is briefly discussed. Here a complete description of such algebra is obtained for the system of difference-difference equations $A_{2}$.

In Section 5 cutting off conditions for the Hirota equation preserving integrability are studied by using the method suggested in [15]. It turned out that some of the systems in the class (1.3) can be obtained from the Hirota equation by imposing proper boundary conditions. Since the cutting off conditions are compatible with the Lax representation one can derive together with the reduced discrete system also its Lax pair. In Section 5 the Lax pairs for the differencedifference systems $A_{N}, B_{N}, C_{N}, A_{1}^{(1)}, D_{N}^{(2)}$ are found.

In Section 6 an algorithm is suggested to look for integrals via the Lax pair. In Section 7 widely known periodical reduction of the Hirota equation is discussed. The Lax pair for this system found in [41] is rewritten in terms of the Cartan-Weyl basis for the algebra $A_{N}^{(1)}$. Emphasize that the difference-difference system obtained as a periodical reduction and that given by the formula (1.3) corresponding to $A_{N}^{(1)}$ are not equivalent (see Remark 7.1 below).

\subsection{Integrals and symmetries for quad graph systems}

Consider a system of quad graph equations of general form

$$
H\left(\mathbf{u}_{n, m}, \mathbf{u}_{n+1, m}, \mathbf{u}_{n, m+1}, \mathbf{u}_{n+1, m+1}\right)=0
$$

where $\mathbf{u}=\mathbf{u}_{n, m}$ is a vector-function depending on two integers and ranging on $\mathbb{C}^{N}: \mathbf{u}=$ $\left(u^{1}, u^{2}, \ldots, u^{N}\right)^{T}$. As usually we request that equation (1.6) can be solved with respect to any of the arguments $\mathbf{u}_{n, m}, \mathbf{u}_{n+1, m}, \mathbf{u}_{n, m+1}, \mathbf{u}_{n+1, m+1}$. In other words there exists a set of functions $H^{( \pm 1, \pm 1)}$ such that

$$
\begin{aligned}
& \mathbf{u}_{n+1, m+1}=H^{1,1}\left(\mathbf{u}_{n, m}, \mathbf{u}_{n+1, m}, \mathbf{u}_{n, m+1}\right), \\
& \mathbf{u}_{n-1, m+1}=H^{(-1,1)}\left(\mathbf{u}_{n-1, m}, \mathbf{u}_{n, m}, \mathbf{u}_{n, m+1}\right), \\
& \mathbf{u}_{n+1, m-1}=H^{(1,-1)}\left(\mathbf{u}_{n+1, m}, \mathbf{u}_{n, m}, \mathbf{u}_{n, m-1}\right), \\
& \mathbf{u}_{n-1, m-1}=H^{(-1,-1)}\left(\mathbf{u}_{n-1, m}, \mathbf{u}_{n, m}, \mathbf{u}_{n, m-1}\right) .
\end{aligned}
$$

Define the following standard set of dynamical variables which consists of the variable $\mathbf{u}_{n, m}$ and its shifts $\mathbf{u}_{n+k, m}$ and $\mathbf{u}_{n, m+l}$ where $k, l \in \mathbb{Z}$

$$
S=\left\{\mathbf{u}_{n+k, m}, \mathbf{u}_{n, m+l}: k, l \in \mathbb{Z}\right\} .
$$

By $[\mathbf{u}]$ we denote a finite set of dynamical variables, for instance notation $h=h([\mathbf{u}])$ means that function $h$ depends on a finite number of dynamical variables. Define shift operators $D_{m}, D_{n}$ acting due to the rules

$$
D_{m} f(n, m)=f(n, m+1), \quad D_{n} f(n, m)=f(n+1, m) .
$$

\section{Definition 1.1.}

i) Function $F([\mathbf{u}], n, m)$ (function $I([\mathbf{u}], n, m))$ is called $m$-integral (respectively $n$-integral) of the equation (1.6) if the following identity holds $D_{m} F=F$ (or, $D_{n} I=I$ ) on arbitrary solutions of equation (1.6). 
ii) Integrals of the form $F=F(n)(I=I(m))$ are called trivial.

iii) Equation admitting $N$ non-trivial independent integrals in each direction is called Darboux integrable.

$i v$ ) Set of integrals is called independent if none of them can be expressed through the other integrals and their shifts.

It can be easily proved that $m$-integrals do not depend on the variables $u_{n, m+l}$ with $l \neq 0$ and similarly $n$-integrals do not depend on $u_{n+k, m}$ where $k \neq 0$.

Now formulate a very simple and convenient sufficient condition of complete set of integrals.

Theorem 1.2. Let us given a set of $m$-integrals of the form

$$
I_{(j)}=I_{(j)}\left(n, m, \mathbf{u}, D_{n} \mathbf{u}, D_{n}^{2} \mathbf{u}, \ldots, D_{n}^{\gamma_{j}} \mathbf{u}\right), \quad \gamma_{j} \geq 0, \quad j=1, \ldots, N .
$$

Suppose that for any $j$ and for all $n, m$ at least one of the derivatives $\frac{\partial I_{(j)}}{\partial u^{i}}$ differs from zero and the condition

$$
\operatorname{det}\left(\frac{\partial I_{(j)}}{\partial u^{i}}\right) \neq 0
$$

holds for all $n, m$. Then the integrals constitute a complete set of integrals.

Proof. Suppose in contrary that the set of integrals (1.7) is not independent. Then at least one of the integrals, say for definiteness $I_{(1)}$ is a function of the form

$$
I_{(1)}=Q\left(I_{(2)}, I_{(3)}, \ldots, I_{(N)}, D_{n} I_{(2)}, D_{n} I_{(3)}, \ldots, D_{n} I_{(N)}, \ldots\right) \text {, }
$$

depending on the other integrals and their shifts. Differentiating (1.9) with respect to $u^{i}$ we obtain the following equalities

$$
\frac{\partial I_{(1)}}{\partial u^{i}}-\frac{\partial I_{(2)}}{\partial u^{i}} \frac{\partial Q}{\partial I_{(2)}}-\cdots-\frac{\partial I_{(N)}}{\partial u^{i}} \frac{\partial Q}{\partial I_{(N)}}=0 .
$$

The latter can be regarded as a linear algebraic system with the coefficient matrix $\left\{\frac{\partial I_{(j)}}{\partial u^{i}}\right\}$ and a solution $\left(1, \frac{\partial Q}{\partial I_{(2)}}, \ldots, \frac{\partial Q}{\partial I_{(N)}}\right)$ which obviously is not trivial. Therefore according to the wellknown theorem the determinant of the coefficient matrix should vanish. But it contradicts (1.8). Thus our assumption that the set of integrals is dependent is not correct. Theorem is proved.

Remark 1.3. Condition (1.8) actually means that jacobian of the map $\mathbf{u} \rightarrow \mathbf{I}=\left(I_{(1)}, \ldots, I_{(N)}\right)$ differs from zero for any value of the variables $\mathbf{u}, \mathbf{D}_{\mathbf{n}} \mathbf{u}, \mathbf{D}_{\mathbf{n}}^{\mathbf{2}} \mathbf{u}, \ldots, \mathbf{D}_{\mathbf{n}}^{\gamma} \mathbf{u}$ ranging in a domain. Here $\gamma=\max \left\{\gamma^{j}\right\}$. Note that (1.8) is not a necessary condition for independent integrals. In the Example 6.1 we have a pair of independent integrals $I_{(1)}$ and $I_{(2)}$ for which condition (1.8) is violated.

Definition 1.4. An equation of the form

$$
\frac{d}{d t} u_{n, m}=G([\mathbf{u}])
$$

is called generalized symmetry of (1.6) if the following compatibility condition is satisfied

$$
\left.\frac{d}{d t}\left(u_{n+1, m+1}-F^{(1,1)}\right)\right|_{F=0, u_{n, m, t}=G}=0 .
$$


It can be shown that function $G([\mathbf{u}])$ is a solution of linearized equation

$$
\frac{\partial F}{\partial u_{n+1, m+1}} D_{n} D_{m} G+\frac{\partial F}{\partial u_{n+1, m}} D_{n} G+\frac{\partial F}{\partial u_{n, m+1}} D_{m} G+\frac{\partial F}{\partial u_{n, m}} G=0 .
$$

There are systems of the form (1.6) which do not admit evolutionary type symmetries, however they admit symmetries of more complicated structure. We call them hyperbolic type symmetries. Such type symmetries for (1.6) consist of two semi-discrete equations

$$
\frac{d}{d t} \mathbf{u}_{n+1, m}=G\left(\frac{d}{d t} \mathbf{u}_{n, m},[\mathbf{u}]\right), \quad \frac{d}{d t} \mathbf{u}_{n, m+1}=\widetilde{G}\left(\frac{d}{d t} \mathbf{u}_{n, m},[\mathbf{u}]\right) .
$$

Definition 1.5. Pair of equations (1.10) define a hyperbolic type symmetry for (1.6) if equations (1.6), (1.10) constitute a commuting triple, i.e. the following compatibility conditions are satisfied

$$
\frac{d}{d t} F^{(1,1)}=D_{m} G=D_{n} \widetilde{G}
$$

by means of equations (1.6), (1.10).

Hyperbolic type symmetries are constructed in Section 3. They are nothing else but non-local symmetries (see, for instance, [35]) rewritten in a more convenient form. In the other hand side existence of hyperbolic type symmetries (1.10) for equation (1.6) can be regarded as a semidiscrete generalization of the well-known property of consistency around a cube $[1,4,32]$ which is approved to be a criterion of integrability.

\section{Systematic approach to the problem of discretization of the Darboux integrable systems}

The problem of finding integrable discretizations of the integrable partial differential equation is very complicated and not enough studied. The same is true for evaluating the continuum limit for discrete models. In [19] an effective algorithm of discretization (as well as evaluation of the continuum limit) of Darboux integrable equations is suggested based on the integrals. In this section we discuss the essence of the algorithm and apply it to exponential type systems. Note that sets of integrals for the systems corresponding to $A_{2}, B_{2}, G_{2}, D_{3}$ considered in this section as easily proved by applying sufficient condition given in Theorem 1.2 are independent.

\subsection{Explanation of the method with example of Liouville equation}

Its well-known that the famous Liuoville equation $u_{x y}=e^{u}$ admits integrals in both directions: $W=u_{x x}-0.5 u_{x}^{2}$ and $\bar{W}=u_{y y}-0.5 u_{y}^{2}$. Indeed, it is very easy to check that $D_{y} W=0$ and $D_{x} \bar{W}=0$.

Consider now the problem of finding all chains of the form

$$
t_{x}(n+1, x)=f\left(x, t(n, x), t(n+1, x), t_{x}(n, x)\right),
$$

having the function $I=t_{x x}-\frac{1}{2} t_{x}^{2}$ as their $n$-integral. Equality $D_{n} I=I$ implies

$$
f_{x}+f_{t} t_{x}+f_{t_{1}} f+f_{t_{x}} t_{x x}-\frac{1}{2} f^{2}=t_{x x}-\frac{1}{2} t_{x}^{2} .
$$

By comparing the coefficients before $t_{x x}$ in $(2.2)$ we have $f_{t_{x}}=1$. Therefore,

$$
f\left(x, t, t_{1}, t_{x}\right)=t_{x}+d\left(x, t, t_{1}\right) .
$$


We substitute (2.3) into (2.2) and get $d_{x}+d_{t} t_{x}+d_{t_{1}} t_{x}+d_{t_{1}} d-\frac{1}{2} t_{x}^{2}-d t_{x}-\frac{1}{2} d^{2}=-\frac{1}{2} t_{x}^{2}$, or equivalently, $d_{t}+d_{t_{1}}-d=0$ and $d_{x}+d_{t_{1}} d-\frac{1}{2} d^{2}=0$. We solve the last two equations simultaneously and find that $d=C e^{\frac{1}{2}\left(t_{1}+t\right)}$ and $C$ is an arbitrary constant. Therefore, chain (2.1) with $n$-integral $I=t_{x x}-\frac{1}{2} t_{x}^{2}$ becomes $t_{1 x}=t_{x}+C e^{\left(t_{1}+t\right) / 2}$. The last equation in its turn admits also an $x$-integral $F=e^{\left(t_{1}-t\right) / 2}+e^{\left(t_{1}-t_{2}\right) / 2}$.

The next step consists in describing equations of the form

$$
v(n+1, m+1)=f(v(n, m), v(n+1, m), v(n, m+1))
$$

with $m$-integral $F=e^{v_{1}-v}+e^{v_{1}-v_{2}}$. Denote $w_{i j}:=e^{-v_{i j}}$. In the new variables $F=\frac{w_{0,0}+w_{2,0}}{w_{1,0}}$ is an $m$-integral of equation $w_{1,1}=g\left(w_{0,0}, w_{1,0}, w_{0,1}\right) . D_{m} F=F$ implies

$$
\frac{w_{2,0}+w_{0,0}}{w_{1,0}}=\frac{D_{n} g+w_{0,1}}{g}
$$

We differentiate both sides of (2.5) with respect to $w_{2,0}$ and apply the shift operator $D_{n}^{-1}$, we have

$$
\frac{1}{w_{1,0}}=\frac{\partial_{w_{2,0}} D_{n} g}{g} \Rightarrow D_{n}^{-1}\left(\frac{1}{w_{1,0}}\right)=D_{n}^{-1}\left(\frac{\partial_{w_{2,0}} D_{n} g}{g}\right) \Rightarrow g_{w_{1,0}}=\frac{w_{0,1}}{w_{0,0}} .
$$

Therefore,

$$
g=\frac{w_{0,1} w_{1,0}}{w_{0,0}}+c\left(w_{0,0}, w_{0,1}\right)
$$

We substitute (2.6) into (2.5) and get

$$
g \frac{w_{0,0}}{w_{1,0}}=c\left(w_{1,0}, g\right)+w_{0,1}
$$

Substitution of (2.6) into (2.7) implies that $c\left(w_{0,0}, w_{0,1}\right) w=c\left(w_{1,0}, g\right) w_{1,0}$, or the same, $c\left(w_{0,0}, w_{0,1}\right) w=D_{n}\left(c\left(w_{0,0}, w_{0,1}\right) w_{0,0}\right)$. Suppose that equation $w_{1,1}=g\left(w, w_{1,0}, w_{0,1}\right)$ does not admit an $m$-integral of the first order, then $c\left(w_{0,0}, w_{0,1}\right) w_{0,0}=D_{n}\left(c\left(w_{0,0}, w_{0,1}\right) w_{0,0}\right)=C=$ const. Thus, $c\left(w_{0,0}, w_{0,1}\right)=C / w_{0,0}$. Finally, $g\left(w_{0,0}, w_{1,0}, w_{0,1}\right)=\frac{w_{0,1} w_{1,0}}{w_{0,0}}+\frac{C}{w_{0,0}}$. Therefore, the equation (2.4) searched with $m$-integral $F=e^{v_{1,0}-v_{0,0}}+e^{v_{1,0}-v_{2,0}}$ becomes $e^{-v_{1,1}-v_{0,0}}=$ $C+e^{-v_{1,0}-v_{0,1}}$, where $C$ is an arbitrary constant. Note that this equation is symmetric with respect to variables $v_{1,0}$ and $v_{0,1}$. Therefore, $n$-integral for the equation can be obtained by simply changing in $m$-integral variables $v_{j, 0}$ into variables $v_{0, j}, j=1,2$.

\subsection{Application of the algorithm of discretization to the system corresponding to the algebra $B_{2}$}

Apply the reasonings above to the system (1.1) corresponding to the algebra $B_{2}$. The first step has already been done in [18], where the differential-difference system was found

$$
u_{1, x}^{1}-u_{x}^{1}=e^{u^{1}+u_{1}^{1}-u_{1}^{2}}, \quad u_{1, x}^{2}-u_{x}^{2}=e^{-2 u^{1}+u^{2}+u_{1}^{2}},
$$

admitting two independent $n$-integrals having the form

$$
\begin{aligned}
I_{(1)}= & 2 u_{x x}^{1}+u_{x x}^{2}-2\left(u_{x}^{1}\right)^{2}+2 u_{x}^{1} u_{x}^{2}-\left(u_{x x}^{2}\right)^{2}, \\
I_{(2)}= & u_{x x x x}^{1}+u_{x}^{1}\left(u_{x x x}^{2}-2 u_{x x x}^{1}\right)+u_{x x}^{1}\left(4 u_{x}^{1} u_{x}^{2}-2\left(u_{x}^{1}\right)^{2}-\left(u_{x}^{2}\right)^{2}\right) \\
& +u_{x x}^{1}\left(u_{x x}^{2}-u_{x x}^{1}\right)+u_{x x}^{2} u_{x}^{1}\left(u_{x}^{1}-2 u_{x}^{2}\right)+\left(u_{x}^{1}\right)^{4}+\left(u_{x}^{1}\right)^{2}\left(u_{x}^{2}\right)^{2}-2\left(u_{x}^{1}\right)^{2} u_{x}^{2},
\end{aligned}
$$


which are also integrals of fully continuous system (1.1) corresponding to the same algebra. It was shown in [18] that system (2.8) admits also $x$-integrals

$$
\begin{aligned}
& F_{(1)}=e^{-u_{0}^{1}+u_{1}^{1}}+e^{-u_{1}^{1}+u_{2}^{1}+u_{2}^{2}-u_{3}^{2}}+e^{u_{1}^{1}-u_{2}^{1}-u_{1}^{2}+u_{2}^{2}}+e^{u_{2}^{1}-u_{3}^{1}} \\
& F_{(2)}=e^{-u_{0}^{2}+u_{1}^{2}}+e^{-2 u_{0}^{1}+2 u_{1}^{1}+u_{1}^{2}-u_{2}^{2}}+2 e^{-u_{0}^{1}+2 u_{1}^{1}-u_{2}^{1}}+e^{2 u_{1}^{1}-2 u_{2}^{1}-u_{1}^{2}+u_{2}^{2}}+e^{u_{2}^{2}-u_{3}^{2}} .
\end{aligned}
$$

The goal of this section is to construct a difference-difference system having the same functions $F_{(1)}$ and $F_{(2)}$ as their $m$-integrals.

To make the formulas shorter we change the variables

$$
a=e^{-u^{1}}, \quad b=e^{-u^{2}}
$$

where $a, b$ are new unknowns.

The given $m$-integrals in these variables read

$$
F_{(1)}=\frac{a_{0}}{a_{1}}+\frac{a_{1} b_{3}}{a_{2} b_{2}}+\frac{a_{2} b_{1}}{a_{1} b_{2}}+\frac{a_{3}}{a_{2}}, \quad F_{(2)}=\frac{b_{0}}{b_{1}}+\frac{a_{0}^{2} b_{2}}{a_{1}^{2} b_{1}}+2 \frac{a_{0} a_{2}}{a_{1}^{2}}+\frac{a_{2}^{2} b_{1}}{a_{1}^{2} b_{2}}+\frac{b_{3}}{b_{2}} .
$$

Formulas (2.9) define integrals for a semi-discrete system (2.8) while (2.11) defines integrals for a certain difference-difference system, that is why the variables in (2.11) should be labeled by a double index, however we omitted here and below in this section the second index, because its value is zero for all considered variables. Now we will look for the equations desired in the variables $a, b$ in the form

$$
a_{1,1}=f\left(a_{0,0}, a_{1,0}, a_{0,1}, b_{0,0}, b_{1,0}, b_{0,1}\right), \quad b_{1,1}=g\left(a_{0,0}, a_{1,0}, a_{0,1}, b_{0,0}, b_{1,0}, b_{0,1}\right) .
$$

Substitute the integrals (2.11) into the equations for $m$-integrals $D_{m} F_{(1)}=F_{(1)}, D_{m} F_{(2)}=F_{(2)}$ and bring them to the following form

$$
\begin{aligned}
& \frac{a_{0,1}}{f}+\frac{f D_{n}^{2} g}{D_{n} f D_{n} g}+\frac{g D_{n} f}{f D_{n} g}+\frac{D_{n}^{2} f}{D_{n} f}=\frac{a_{0}}{a_{1}}+\frac{a_{1} b_{3}}{a_{2} b_{2}}+\frac{a_{2} b_{1}}{a_{1} b_{2}}+\frac{a_{3}}{a_{2}}, \\
& \frac{b_{0,1}}{g}+\frac{a_{0,1} D_{n} g}{f^{2} g}+2 \frac{a_{0,1} D_{n} f}{f^{2}}+\frac{g D_{n} f^{2}}{f^{2} D_{n} g}+\frac{D_{n}^{2} g}{D_{n} g}=\frac{b_{0}}{b_{1}}+\frac{a_{0}^{2} b_{2}}{a_{1}^{2} b_{1}}+2 \frac{a_{0} a_{2}}{a_{1}{ }^{2}}+\frac{a_{2}^{2} b_{1}}{a_{1}^{2} b_{2}}+\frac{b_{3}}{b_{2}} .
\end{aligned}
$$

By differentiating equation $(2.12 \mathrm{~b})$ with respect to the variables $a_{3}, b_{3}$ we get equations

$$
\frac{1}{D_{n} g} D_{n}^{2} \frac{\partial g}{\partial a_{1}}=0 \Leftrightarrow \frac{\partial g}{\partial a_{1}}=0, \quad \frac{1}{D_{n} g} D_{n}^{2} \frac{\partial g}{\partial b_{1}}=\frac{1}{b_{2}} \Leftrightarrow \frac{\partial g}{\partial b_{1}}=\frac{b_{0,1}}{b_{0}},
$$

which imply immediately

$$
g\left(a_{0,0}, a_{1,0}, a_{0,1}, b_{0,0}, b_{1,0}, b_{0,1}\right)=\frac{b_{1,0} b_{0,1}}{b_{0,0}}+\frac{g_{1}\left(a_{0,0}, a_{0,1}, b_{0,0}, b_{0,1}\right)}{b_{0,0}} .
$$

Similarly, differentiation of (2.12a) with respect to $a_{3}, b_{3}$ yields

$$
\begin{aligned}
& \frac{1}{D_{n} f} D_{n}^{2} \frac{\partial f}{\partial a_{1}}=\frac{1}{a_{2}} \Leftrightarrow \frac{\partial f}{\partial a_{1}}=\frac{a_{0,1}}{a_{0}}, \\
& \frac{f D_{n} g}{b_{2} D_{n} f D_{n} g}+\frac{1}{D_{n} f} D_{n}^{2} \frac{\partial f}{\partial b_{1}}=\frac{a_{1}}{a_{2} b_{2}} \Leftrightarrow \frac{\partial f}{\partial b_{1}}=\frac{1}{a_{0} b_{0}} D_{n}^{-1}\left(a_{0} f-a_{1} a_{0,1}\right) .
\end{aligned}
$$

From these equations we get

$$
f\left(a_{0,0}, a_{1,0}, a_{0,1}, b_{0,0}, b_{1,0}, b_{0,1}\right)=\frac{a_{1,0} a_{0,1}}{a_{0,0}}+\frac{C b_{1,0}}{a_{0,0}} .
$$


By differentiating (2.12) with respect to $a_{2}$ we obtain

$$
\begin{aligned}
& \frac{a_{1,1}}{b_{2} b_{2,1}} \frac{\partial}{\partial a_{2}} \frac{D_{n}^{2} g_{1}}{a_{2,1}}+\frac{b_{1,1}}{a_{1} b_{2,1}}=\frac{b_{1}}{a_{1} b_{2}}, \\
& 2 \frac{a_{0,1}}{a_{1,1} a_{1}}+2 \frac{a_{2,1} b_{1,1}}{a_{1} a_{1,1} b_{2,1}}+\frac{1}{b_{2,1} b_{2,0}} \frac{\partial}{\partial a_{2}} D_{n}^{2} g_{1}=2 \frac{a_{0}}{a_{1}}+2 \frac{a_{2} b_{1}}{a_{1}^{2} b_{2}} .
\end{aligned}
$$

The last two equations can be rewritten as follows

$$
\frac{\partial}{\partial a_{1}} \frac{D_{n} g_{1}}{a_{1,1}}=\frac{g_{1}}{a_{0} a_{0,1}}, \quad \frac{\partial}{\partial a_{1}} D_{n} g_{1}=2 \frac{a_{1,1} g_{1}}{a_{0,1} a_{0}} .
$$

By getting rid of the variable $\partial_{a_{1}} D_{n} g_{1}$ in these equations we find

$$
\frac{D_{n} g_{1}}{a_{1,1}^{2}}=\frac{g_{1}}{a_{0,1}^{2}} \Leftrightarrow g_{1}=C_{1} a_{0,1}^{2}
$$

The last implication is due to the assumption that $F_{(1)}$ and $F_{(2)}$ are integrals of the lowest order.

As a result we get equations

$$
a_{1,1} a_{0,0}-a_{1,0} a_{0,1}=C b_{1,0}, \quad b_{1,1} b_{0,0}-b_{1,0} b_{0,1}=C_{1} a_{0,1}^{2} .
$$

After some rescaling we can put $C=C_{1}=1$. Finally, we end up with the system of discrete equations

$$
a_{1,1} a_{0,0}-a_{1,0} a_{0,1}=b_{1,0}, \quad b_{1,1} b_{0,0}-b_{1,0} b_{0,1}=a_{0,1}^{2} .
$$

corresponding to the algebra $B_{2}$. Turn back to the original variables $u^{1}, u^{2}$ (see $(2.10)$ )

$$
\Delta u^{1}=e^{u_{0,1}^{1}+u_{1,0}^{1}-u_{1,0}^{2}}, \quad \Delta u^{2}=e^{-2 u_{0,1}^{1}+u_{0,1}^{2}+u_{1,0}^{2}} .
$$

By construction the last system admits pair of integrals (2.9) in which the second index for the variables $u^{1}, u^{2}$ is omitted, since its value is the same for all variables. To find the integrals in the other direction one uses the discrete symmetry $n \leftrightarrow-m$ of the system.

\subsection{Integrals of the systems corresponding to the algebras $A_{2}, G_{2}, D_{3}$}

\subsubsection{System corresponding to the algebra $A_{2}$}

The Cartan matrix of $A_{2}$ is

$$
A=\left(\begin{array}{cc}
2 & -1 \\
-1 & 2
\end{array}\right)
$$

The discrete system for $A_{2}$ looks as follows

$$
\Delta\left(u^{1}\right)=e^{u_{0,1}^{1}+u_{1,0}^{1}-u_{1,0}^{2}}, \quad \Delta\left(u^{2}\right)=e^{-u_{0,1}^{1}+u_{0,1}^{2}+u_{1,0}^{2}} .
$$

For system (2.13) $m$-integrals are

$$
\begin{aligned}
& F_{(1)}=e^{-u_{0,0}^{2}+u_{1,0}^{2}}+e^{-u_{0,0}^{1}+u_{1,0}^{1}+u_{1,0}^{2}-u_{2,0}^{2}}+e^{u_{1,0}^{1}-u_{2,0}^{1}}, \\
& F_{(2)}=e^{-u_{0,0}^{1}+u_{1,0}^{1}}+e^{u_{1,0}^{1}-u_{2,0}^{1}-u_{1,0}^{2}+u_{2,0}^{2}}+e^{u_{2,0}^{2}-u_{3,0}^{2}}
\end{aligned}
$$

To find the integrals in the other direction one uses the discrete symmetry $n \leftrightarrow-m$ of the system. 


\subsubsection{System corresponding to the algebra $G_{2}$}

The Cartan matrix of $G_{2}$ is

$$
A=\left(\begin{array}{cc}
2 & -1 \\
-3 & 2
\end{array}\right)
$$

The discrete system for $G_{2}$ is of the form

$$
\Delta\left(u^{1}\right)=e^{u_{0,1}^{1}+u_{1,0}^{1}-u_{1,0}^{2}}, \quad \Delta\left(u^{2}\right)=e^{-3 u_{0,1}^{1}+u_{0,1}^{2}+u_{1,0}^{2}} .
$$

Its $m$-integrals are

$$
\begin{aligned}
F_{(1)}= & e^{u_{1,0}^{1}-u_{2,0}^{1}}+e^{u_{-1,0}^{1}-u_{-2,0}^{1}}+e^{u_{1,0}^{2}+u_{1,0}^{1}-u_{1,0}^{1}-u_{2,0}^{2}}+e^{u_{-1,0}^{1}+u_{0,0}^{2}-u_{0,0}^{1}-u_{-1,0}^{2}} \\
& +e^{u_{1,0}^{2}+2 u_{0,0}^{1}-u_{0,0}^{2}-2 u_{1,0}^{1}}+e^{u_{0,0}^{2}+2 u_{0,0}^{1}-u_{1,0}^{2}-2 u_{-1,0}^{1}}+2 e^{2 u_{0,0}^{1}-u_{-1,0}^{1}-u_{1,0}^{1}}, \\
F_{(2)}= & e^{u_{2,0}^{2}-u_{3,0}^{2}}+e^{3 u_{0,0}^{1}+u_{0,0}^{2}-u_{1,0}^{2}-3 u_{-1,0}^{1}}+3 e^{u_{0,0}^{1}+u_{1,0}^{2}-u_{2,0}^{1}-u_{0,0}^{2}}+3 e^{u_{0,0}^{1}+u_{1,0}^{1}-u_{-1,0}^{1}-u_{2,0}^{1}} \\
& +3 e^{u_{1,0}^{2}+3 u_{1,0}^{1}-2 u_{0,0}^{1}-u_{2,0}^{2}-u_{2,0}^{1}}+3 e^{3 u_{1,0}^{1}+u_{0,0}^{1}-2 u_{2,0}^{1}}+3 e^{3 u_{0,0}^{1}+u_{1,0}^{1}-u_{-1,0}^{1}} \\
& +3 e^{3 u_{0,0}^{1}+u_{1,0}^{2}-u_{0,0}^{2}-2 u_{1,0}^{1}-u_{-1,0}^{1}}+3 e^{u_{1,0}^{2}+u_{1,0}^{2}-u_{2,0}^{2}-u_{-1,0}^{1}}+2 e^{2 u_{1,0}^{2}+u_{0,0}^{2}-u_{2,0}^{2}} \\
& +e^{3 u_{1,0}^{1}+u_{2,0}^{2}-u_{1,0}^{2}-3 u_{2,0}^{1}}+e^{3 u_{1,0}^{1}+2 u_{1,0}^{2}-3 u_{0,0}^{1}-2 u_{2,0}^{2}}+e^{3 u_{0,0}^{1}+2 u_{1,0}^{2}-2 u_{0,0}^{2}-3 u_{1,0}^{1}}+e^{u_{2,0}^{2}+u_{-1,0}^{2}} .
\end{aligned}
$$

To find the integrals in the other direction one uses the discrete symmetry $n \leftrightarrow-m$ of the system.

\subsubsection{System corresponding to the algebra $D_{3}$}

The Cartan matrix of $D_{3}$ is

$$
A=\left(\begin{array}{ccc}
2 & -1 & -1 \\
-1 & 2 & 0 \\
-1 & 0 & 2
\end{array}\right)
$$

The discrete system for $D_{3}$ looks as

$$
\Delta\left(u^{1}\right)=e^{u_{0,1}^{1}+u_{1,0}^{1}-u_{0,1}^{2}-u_{0,1}^{3},} \quad \Delta\left(u^{2}\right)=e^{u_{0,1}^{2}+u_{1,0}^{2}-u_{1,0}^{1}}, \quad \Delta\left(u^{3}\right)=e^{u_{0,1}^{3}+u_{1,0}^{3}-u_{1,0}^{1}} .
$$

Its $m$-integrals are

$$
\begin{aligned}
F_{(1)}= & e^{u_{0,0}^{3}-u_{-1,0}^{3}}+e^{u_{1,0}^{2}-u_{2,0}^{2}}+e^{u_{1,0}^{2}+u_{1,0}^{1}-u_{2,0}^{1}-u_{0,0}^{2}}+e^{u_{1,0}^{1}+u_{0,0}^{3}-u_{0,0}^{1}-u_{1,0}^{3}} \\
F_{(2)}= & e^{u_{0,0}^{2}-u_{-1,0}^{2}}+e^{u_{1,0}^{3}-u_{2,0}^{3}}+e^{u_{1,0}^{3}+u_{1,0}^{1}-u_{2,0}^{1}-u_{0,0}^{3}}+e^{u_{1,0}^{1}+u_{0,0}^{2}-u_{0,0}^{1}-u_{1,0}^{2}} \\
F_{(3)}= & e^{u_{1,0}^{1}-u_{2,0}^{1}}+e^{u_{0,0}^{1}-u_{-1,0}^{1}}+e^{u_{0,0}^{2}-u_{1,0}^{2}+u_{0,0}^{3}-u_{-1,0}^{3}}+e^{u_{0,0}^{2}-u_{-1,0}^{2}+u_{0,0}^{3}-u_{1,0}^{3}}+ \\
& +e^{u_{1,0}^{1}-u_{0,0}^{1}+u_{0,0}^{2}-u_{1,0}^{2}+u_{0,0}^{3}-u_{1,0}^{3}}+e^{u_{0,0}^{1}-u_{1,0}^{1}+u_{0,0}^{2}-u_{-1,0}^{2}+u_{0,0}^{3}-u_{-1,0}^{3}}
\end{aligned}
$$

By applying Theorem 1.2 one can prove that these integrals provide a complete set of inde-

pendent integrals. To find the integrals in the other direction one uses the discrete symmetry $n \leftrightarrow-m$ of the system.

\section{Symmetries of discrete systems}

In this section we demonstrate that the discrete systems admit generalized symmetries. For the case of the simple Lie algebras the systems admit local symmetries while for the case of affine algebras the symmetries are nonlocal. 


\subsection{Generalized symmetries for the system $A_{2}$}

Higher symmetries for system corresponding to $A_{2}$ are found by using the method suggested in [12] (see also [26]). The lowest order symmetry is of the form

$$
\frac{d u_{0,0}^{1}}{d t}=\frac{3 e^{u_{0,0}^{1}-u_{1,0}^{1}}}{D_{m}^{-1} F_{(1)}}-1, \quad \frac{d u_{0,0}^{2}}{d t}=1-\frac{3 e^{u_{0,0}^{2}-u_{-1,0}^{2}}}{D_{m}^{-1} F_{(1)}} .
$$

The next order symmetry depends on arbitrary functions $f(x, y), g(x)$ of two and respectively one variable and has the form

$$
\begin{aligned}
\frac{d u_{0,0}^{1}}{d t}= & \frac{f\left(F_{(1)}, D_{m}^{-1} F_{(2)}\right)+g\left(D_{m}^{-1} F_{(1)}\right)}{D_{m}^{-1} F_{(2)}} e^{u_{0,0}^{1}-u_{-1,0}^{1}+f\left(F_{(1)}, D_{m}^{-1} F_{(2)}\right)-g\left(F_{(1)}\right)} \\
& +g\left(D_{m}^{-1} F_{(1)}\right)-\frac{f\left(F_{(1)}, D_{m}^{-1} F_{(2)}\right)+f\left(D_{m}^{-2} F_{(1)}, D_{m}^{-2} F_{(2)}\right)-g\left(F_{(1)}\right)}{D_{m}^{-1} F_{(1)}} e^{u_{0,0}^{1}-u_{1,0}^{1},}, \\
\frac{d u_{0,0}^{2}}{d t}= & \frac{f\left(F_{(1)}, D_{m}^{-1} F_{(2)}\right)+f\left(D_{m}^{-2} F_{(1)}, D_{m}^{-2} F_{(2)}\right)-g\left(F_{(1)}\right)}{D_{m}^{-1} F_{(1)}} e^{u_{0,0}^{2}-u_{-1,0}^{2}} \\
& -f\left(D_{m}^{-2} F_{(1)}, D_{m}^{-2} F_{(2)}\right)+\frac{f\left(D_{m}^{-1} F_{(1)}, D_{m}^{-2} F_{(2)}\right)+g\left(D_{m}^{-2} F_{(1)}\right)}{D_{m}^{-2} F_{(2)}} e^{u_{0,0}^{2}-u_{1,0}^{2}} .
\end{aligned}
$$

\subsection{Evaluation of hyperbolic type symmetries for the system $A_{1}^{(1)}$}

The method for searching nonlocal symmetries is illustrated with the following example

$$
e^{-u_{1,1}-u_{0,0}}-e^{-u_{1,0}-u_{0,1}}=e^{-2 v_{1,0}}, \quad e^{-v_{1,1}-v_{0,0}}-e^{-v_{1,0}-v_{0,1}}=e^{-2 u_{0,1}},
$$

corresponding to the algebra $A_{1}^{(1)}$ with the Cartan matrix

$$
A=\left(\begin{array}{cc}
2 & -2 \\
-2 & 2
\end{array}\right)
$$

Recall that the continuous version of the system is

$$
u_{x y}=\exp (2 u-2 v), \quad v_{x y}=\exp (2 v-2 u) .
$$

It can be proved that system (3.2) does not have any local generalized symmetry, however it has nonlocal generalized symmetries, the simplest one can be represented in terms of $s=u+v$, $r=u-v$ as follows

$$
r_{t}=r_{x x x}-2 r_{x}^{3}, \quad s_{x t}=2 r_{x} r_{x x x}-3 r_{x}^{4}-r_{x x}^{2}+F\left(W, W_{x}\right) .
$$

Here $F$ is an arbitrary function and $W=s_{x x}-r_{x}^{2}$ is the $y$-integral of system (3.2). Note that years ago in [9] it was observed that equation $r_{t}=r_{x x x}-2 r_{x}^{3}$ is consistent with the system (3.2).

For the fully discrete analogue (3.1) of the system (3.2) we have a very similar situation. The system (3.1) does not have any local generalized symmetry. One has to look for a nonlocal symmetry. The main trouble arising in this case is connected with the guessing of the structure of non-locality and the form of the symmetry.

Rewrite system (3.1) in the form

$$
a_{1,1} a_{0,0}-a_{1,0} a_{0,1}=b_{1,0}^{2}, \quad b_{1,1} b_{0,0}-b_{1,0} b_{0,1}=a_{0,1}^{2},
$$

where $a_{i, j}=\exp \left(-u_{i, j}\right), b_{i, j}=\exp \left(-v_{i, j}\right)$. 
We will look for the hyperbolic type symmetry (see Definition 1.5) of (3.3) in such a form

$$
\begin{aligned}
& \partial_{t} a_{1,0}=f_{1}\left(a_{0,0}, a_{1,0}\right) \partial_{t} a_{0,0}+f\left(a_{-1,0}, a_{0,0}, a_{1,0}, a_{2,0}, b_{-1,0}, b_{0,0}, b_{1,0}, b_{2,0}\right), \\
& \partial_{t} b_{1,0}=g_{1}\left(b_{0,0}, b_{1,0}\right) \partial_{t} b_{0,0}+g\left(a_{-1,0}, a_{0,0}, a_{1,0}, a_{2,0}, b_{-1,0}, b_{0,0}, b_{1,0}, b_{2,0}\right) .
\end{aligned}
$$

Here $f_{1}, g_{1}, f, g$ are unknown functions. Right hand side of (3.4) corresponds to the function $G$ in (1.10). The function $\widetilde{G}$ is evaluated in terms of $G$ below by means of the compatibility conditions.

From (3.4) we can find

$$
\partial_{t} a_{1,1}=D_{m}\left(f_{1}\right) \partial_{t} a_{0,1}+D_{m} f, \quad \partial_{t} b_{1,1}=D_{m}\left(g_{1}\right) \partial_{t} b_{0,1}+D_{m} g
$$

After differentiation (3.1) with respect to $t$ by means of the systems (3.4), (3.5) we get

$$
\begin{aligned}
& \partial_{t} a_{0,0}\left(a_{1,1}-a_{0,1} f_{1}\right)+\partial_{t} a_{0,1}\left(a_{0,0} D_{m} f_{1}-a_{1,0}\right)-2 \partial_{t} b_{0,0} b_{1,0} g_{1}=\cdots, \\
& -2 a_{0,1} \partial_{t} a_{0,1}+\partial_{t} b_{0,0}\left(b_{1,1}-b_{0,1} g_{1}\right)+\partial_{t} b_{0,1}\left(b_{0,0} D_{m} g_{1}-b_{1,0}\right)=\cdots
\end{aligned}
$$

Here the right hand sides do not depend on the derivatives of dynamical variables with respect to $t$. By applying the operator $D_{n}$ to both sides of (3.6) we obtain

$$
\begin{aligned}
& \partial_{t} a_{0,0} f_{1} D_{n}\left(a_{1,1}-a_{0,1} f_{1}\right)+\partial_{t} a_{0,1} D_{m} f_{1} D_{n}\left(a_{0,0} D_{m} f_{1}-a_{1,0}\right)-2 \partial_{t} b_{1,0} b_{2,0} g_{1} D_{n} g_{1}=\cdots, \\
& -2 a_{1,1} \partial_{t} D_{m} f_{1}+\partial_{t} b_{0,0} g_{1} D_{n}\left(b_{1,1}-b_{0,1} g_{1}\right)+\partial_{t} b_{0,1} D_{m} g_{1} D_{n}\left(b_{0,0} D_{m} g_{1}-b_{1,0}\right)=\cdots
\end{aligned}
$$

System of equations (3.6), (3.7) is a system of linear algebraic equations with unknowns $\partial_{t} a_{0,0}$, $\partial_{t} b_{0,0}, \partial_{t} a_{0,1}, \partial_{t} b_{0,1}$. If the determinant of this system is different from zero, then due to the Cramer's rule the system has unique solution, and therefore the searched generalized symmetry is local. This output is in contradiction with our previous study proving the absence of local symmetries. Thus the determinant should be zero

$$
\begin{aligned}
& \mid \begin{array}{cc}
a_{1,1}-f_{1} a_{0,1} & a_{0,0} D_{m} f_{1}-a_{1,0} \\
f_{1}\left(a_{2,1}-a_{1,1} D_{n} f_{1}\right) & \left(a_{1,0} D_{n} D_{m} f_{1}-a_{2,0}\right) D_{m} f_{1} \\
0 & -2 a_{1,1} D_{m} f_{1} \\
0 & -2 a_{0,1}
\end{array} \\
& \begin{array}{cc}
-2 b_{1,0} g_{1} & 0 \\
-2 b_{2,0} g_{1} D_{n} g_{1} & 0 \\
g_{1}\left(b_{2,1}-b_{1,1} D_{n} g_{1}\right) & D_{m} g_{1}\left(b_{1,0} D_{n} D_{m} g_{1}-b_{2,0}\right) \\
b_{1,1}-g_{1} b_{0,1} & b_{0,0} D_{m} g_{1}-b_{1,0}
\end{array} \mid
\end{aligned}
$$

Vanishing of the determinant implies

$$
f_{1}\left(a_{0,0}, a_{1,0}\right)=a_{1,0} / a_{0,0}, \quad g_{1}\left(b_{0,0}, b_{1,0}\right)=b_{1,0} / b_{0,0},
$$

In virtue of (3.8) equations (3.6), (3.7) take the form

$$
\begin{aligned}
& \frac{\partial_{t} a_{0,0}}{a_{0,0}}+\frac{\partial_{t} a_{0,1}}{a_{0,1}}-2 \frac{\partial_{t} b_{0,0}}{b_{0,0}}=\frac{2}{b_{1,0}} g+\frac{a_{0,1}}{b_{1,0}^{2}} f-\frac{a_{0,0}}{b_{1,0}^{2}} D_{m} f, \\
& \frac{\partial_{t} a_{0,0}}{a_{0,0}}+\frac{\partial_{t} a_{0,1}}{a_{0,1}}-2 \frac{\partial_{t} b_{0,0}}{b_{0,0}}=\frac{2}{b_{2,0}} D_{n} g+\frac{a_{1,1}}{b_{2,0}^{2}} D_{n} f-\frac{a_{1,0}}{b_{2,0}^{2}} D_{n} D_{m} f-\frac{f}{a_{1,0}}-\frac{D_{m} f}{a_{1,1}}+\frac{2}{b_{1,0}} g, \\
& \frac{\partial_{t} b_{0,0}}{b_{0,0}}+\frac{\partial_{t} b_{0,1}}{b_{0,1}}-2 \frac{\partial_{t} a_{0,1}}{a_{0,1}}=\frac{b_{0,1}}{a_{0,1}^{2}} g+\frac{b_{0,0}}{a_{0,1}^{2}} D_{m} g, \\
& \frac{\partial_{t} b_{0,0}}{b_{0,0}}+\frac{\partial_{t} b_{0,1}}{b_{0,1}}-2 \frac{\partial_{t} a_{0,1}}{a_{0,1}}=\frac{b_{1,1}}{a_{1,1}^{2}} D_{n} g+\frac{b_{1,0}}{a_{1,1}^{2}} D_{n} D_{m} g+\frac{2}{a_{1,1}} D_{m} f-\frac{g}{b_{1,0}}-\frac{D_{m} g}{b_{1,1}} .
\end{aligned}
$$


We can see that the left hand sides of the first and third equations as well as the left hand sides of the second and fourth equations of the last system are identical. Comparison of their right hand sides gives a system of two equation for $f, g$

$$
\begin{aligned}
& \frac{a_{1,0}}{b_{2,0}^{2}} D_{n} D_{m} f-\frac{a_{1,1}}{b_{2,0}^{2}} D_{n} f-\frac{a_{1,0} a_{0,1}}{a_{1,1} b_{1,0}^{2}} D_{m} f+\frac{a_{1,1} a_{0,0}}{a_{1,0} b_{1,0}^{2}} f-\frac{2}{b_{2,0}} D_{n} g=0, \\
& \frac{b_{1,0}}{a_{1,1}^{2}} D_{n} D_{m} g+\frac{b_{1,1}}{a_{1,1}^{2}} D_{n} g-\frac{b_{1,0} b_{0,1}}{b_{1,1} a_{0,1}^{2}} D_{m} g-\frac{b_{1,1} b_{0,0}}{b_{1,0} a_{0,1}^{2}} g+\frac{2}{a_{1,1}} D_{m} f=0 .
\end{aligned}
$$

Differentiate (3.10) and (3.11) with respect to $a_{3,0}, b_{3,0}, b_{-1,0}$ or $a_{-1,0}$ and get

$$
\begin{aligned}
& \frac{b_{0,0} a_{0,0}}{a_{1,0}^{2}} \frac{\partial f}{\partial b_{-1,0}}=D_{m}\left(\frac{b_{0,0} a_{0,0}}{a_{1,0}^{2}} \frac{\partial f}{\partial b_{-1,0}}\right) \\
& D_{m}\left(\frac{a_{0,0}^{2}}{a_{1,0}^{2}} \frac{\partial f}{\partial a_{-1,0}}\right)=\frac{a_{0,0}^{2}}{a_{1,0}^{2}} \frac{\partial f}{\partial a_{-1,0}}+\frac{2 a_{-1,1} a_{0,1}^{2}}{a_{1,1}^{2} b_{0,0}} D_{m} \frac{\partial f}{\partial b_{-1,0}}, \\
& \frac{a_{1,0}}{b_{0,0}} \frac{\partial g}{\partial a_{2,0}}=\frac{a_{1,1}}{b_{0,1}} D_{m} \frac{\partial g}{\partial a_{2,0}}, \quad D_{m} \frac{\partial g}{\partial b_{2,0}}+\frac{2 b_{1,0} b_{2,0}}{a_{1,0} b_{1,1}} D_{m} \frac{\partial g}{\partial a_{2,0}}=\frac{b_{1,0} b_{0,1}}{b_{0,0} b_{1,1}} \frac{\partial g}{\partial b_{2,0}}, \\
& \frac{\partial f}{\partial a_{2,0}}+\frac{2 b_{1,0}}{a_{0,1}} \frac{\partial g}{\partial a_{2,0}}=\frac{a_{1,1} a_{0,0}}{a_{1,0} a_{0,1}} D_{m} \frac{\partial f}{\partial a_{2,0}}, \\
& \frac{\partial f}{\partial b_{2,0}}+\frac{2 b_{1,0}}{a_{0,1}} \frac{\partial g}{\partial b_{2,0}}=\frac{2 a_{0,0} b_{2,0}}{a_{1,0} a_{0,1}} D_{m} \frac{\partial f}{\partial a_{2,0}}+\frac{a_{0,0} b_{1,1}}{a_{0,1} b_{1,0}} D_{m} \frac{\partial f}{\partial b_{2,0}} \\
& \frac{\partial g}{\partial b_{-1,0}}+\frac{2 b_{1,0} a_{0,0}^{2}}{a_{1,0} b_{1,-1} b_{0,0}} \frac{\partial f}{\partial b_{-1,0}}=\frac{b_{1,0}^{2} b_{0,-1}^{2}}{b_{1,-1}^{2} b_{00,}^{2}} D_{m}^{-1} \frac{\partial g}{\partial b_{-1,0}}, \\
& \frac{\partial g}{\partial a_{-1,0}}+\frac{2 a_{0,0}^{2} b_{1,0}}{a_{1,0} b_{1,-1} b_{0,0}} \frac{\partial f}{\partial a_{-1,0}}=\frac{b_{1,0}^{2} a_{0,-1} b_{0,-1}}{a_{0,0} b_{0,0} b_{1,-1}} D_{m}^{-1} \frac{\partial g}{\partial a_{-1,0}} \\
& +\frac{4 a_{0,0}^{2} a_{-1,0} b_{1,0}}{a_{1,0} b_{0,-1} b_{1,-1} b_{0,0}} \frac{\partial f}{\partial b_{-1,0}}+\frac{2 a_{-1,0}}{b_{0,-1}} \frac{\partial g}{\partial b_{-1,0}} .
\end{aligned}
$$

We use the last system of eight equations in order to specify dependence of the functions $f, g$ on the variables $a_{-1,0}, a_{2,0}, b_{-1,0}, b_{2,0}$ corresponding to the lowest and highest values of the first index. From the first equation in (3.12) we get

$$
\frac{\partial f}{\partial b_{-1,0}}=\frac{C_{7} a_{1,0}^{2}}{b_{0,0} a_{0,0}}
$$

Then the second equation takes the form

$$
D_{m}\left(\frac{a_{0,0}^{2}}{a_{1,0}^{2}} \frac{\partial f}{\partial a_{-1,0}}\right)=\frac{a_{0,0}^{2}}{a_{1,0}^{2}} \frac{\partial f}{\partial a_{-1,0}}+\frac{2 C_{7} a_{-1,1} a_{0,1}}{b_{0,1} b_{0,0}} .
$$

It can easily be proved that the expression $\frac{2 a_{-1,1} a_{0,1}}{b_{0,1} b_{0,0}}$ does not belong to the image of the operator $D_{m}-1$, so we have to put $C_{7}=0$ and then

$$
\frac{\partial f}{\partial a_{-1,0}}=\frac{C_{4} a_{1,0}^{2}}{a_{0,0}^{2}} \text {. }
$$

From the pair of equations (3.13) we have

$$
\frac{\partial g}{\partial a_{2,0}}=0, \quad \frac{\partial g}{\partial b_{2,0}}=\frac{C_{2} b_{0,0}}{b_{1,0}}
$$


Continuing this way one can find dependence of the functions $f, g$ on the variables $a_{-1,0}, a_{2,0}$, $b_{-1,0}, b_{2,0}$

$$
\begin{aligned}
f= & \frac{a_{0,0} b_{2,0}}{b_{1,0}} C_{1}+C_{2}\left(\frac{a_{0,0} a_{2,0}}{a_{1,0}}+\frac{a_{0,0} b_{2,0}^{2}}{a_{1,0} b_{1,0}^{2}}+\frac{2 b_{0,0} a_{1,0} b_{2,0}}{b_{1,0}^{2}}\right) \\
& +\frac{a_{1,0}^{2} a_{-1,0}}{a_{0,0}^{2}} C_{4}+f_{1}\left(a_{0,0}, a_{1,0}, b_{0,0}, b_{1,0}\right), \\
g= & \frac{b_{0,0} b_{2,0}}{b_{1,0}} C_{2}+C_{4}\left(\frac{b_{1,0}^{2} b_{-1,0}}{b_{0,0}^{2}}+\frac{b_{1,0}^{3} a_{-1,0}^{2}}{a_{0,0}^{2} b_{0,0}^{2}}+\frac{2 b_{1,0} a_{1,0} a_{-1,0}}{a_{0,0}^{2}}\right) \\
& +\frac{b_{1,0}^{2} a_{-1,0}}{a_{0,0} b_{0,0}} C_{5}+g_{1}\left(a_{0,0}, a_{1,0}, b_{0,0}, b_{1,0}\right) .
\end{aligned}
$$

For the functions $f_{1}, g_{1}$ from the same system (3.10), (3.11) we obtain the following nonhomogeneous equations

$$
\begin{aligned}
& \frac{a_{1,0}}{b_{2,0}^{2}} D_{n} D_{m} f_{1}-\frac{a_{1,1}}{b_{2,0}^{2}} D_{n} f_{1}-\frac{a_{1,0} a_{0,1}}{a_{1,1} b_{2,0}^{2}} D_{m} f_{1}+\frac{a_{1,1} a_{0,0}}{a_{1,0} b_{1,0}^{2}} f_{1}-\frac{2}{b_{2,0}} D_{n} g_{1}=\cdots \\
& \frac{b_{1,0}}{a_{1,1}^{2}} D_{n} D_{m} g_{1}+\frac{b_{1,1}}{a_{1,1}^{2}} D_{n} g_{1}-\frac{b_{1,0} b_{0,1}}{b_{1,1} a_{0,1}^{2}} D_{m} g_{1}-\frac{b_{1,1} b_{0,0}}{b_{1,0} a_{0,1}^{2}} g_{1}+\frac{2}{a_{1,1}} D_{m} f_{1}=\cdots
\end{aligned}
$$

Differentiating (3.15) with respect to the variable $a_{2,0}$ we get

$$
\frac{a_{0,0}}{b_{0,0}} \frac{\partial g_{1}}{\partial a_{1,0}}-D_{m}\left(\frac{a_{0,0}}{b_{0,0}} \frac{\partial g_{1}}{\partial a_{1,0}}\right)=\frac{2 C_{2}\left(a_{0,1}^{3} a_{1,0}-b_{1,0}^{3} b_{0,1}\right)}{a_{0,0} a_{0,1} b_{1,1} b_{1,0}}+2\left(C_{4}-C_{2}\right) \frac{a_{0,1}^{3} a_{-1,0}-b_{1,1} b_{0,0}^{3}}{a_{0,0} b_{0,0} a_{0,1} b_{0,1}} .
$$

From this equation we can find

$$
C_{4}=C_{2}, \quad \frac{a_{0,0}}{b_{0,0}} \frac{\partial g_{1}}{\partial a_{1,0}}=\frac{2 C_{2} a_{1,0} b_{0,0}}{a_{0,0} b_{1,0}}+C_{6},
$$

therefore

$$
g_{1}=C_{2} \frac{a_{1,0}^{2} b_{0,0}^{2}}{a_{0,0}^{2} b_{1,0}}+C_{6} \frac{a_{1,0} b_{0,0}}{a_{0,0}}+g_{2}\left(a_{0,0}, b_{0,0}, b_{1,0}\right) .
$$

Differentiating (3.15) with respect to $b_{2,0}$ we get

$$
D_{m}\left(\frac{\partial g_{2}}{\partial b_{1,0}}\right)-\frac{\partial g_{2}}{\partial b_{1,0}}=2\left(C_{1}-C_{6}\right) \frac{b_{0,0} b_{1,0}}{a_{0,0} a_{0,1}}+2\left(C_{1}-C_{5}\right) \frac{a_{0,1}^{2} a_{0,0} a_{-1,1}-b_{0,0}^{2} b_{1,0} b_{0,1}}{a_{0,0} b_{0,0} a_{0,1} b_{0,1}},
$$

therefore

$$
C_{6}=C_{1}, \quad C_{5}=C_{1}, \quad g_{2}=C_{3} b_{1,0}+g_{3}\left(a_{0,0}, b_{0,0}\right) .
$$

Now apply the operator $D_{n}^{-1}$ to the equation (3.14) and then differentiate the obtained result with respect to the variable $b_{-1,0}$

$$
D_{m}\left(\frac{a_{0,0} b_{1,0}}{a_{1,0}^{2}} \frac{\partial f_{1}}{\partial b_{0,0}}\right)-\frac{a_{0,0} b_{1,0}}{a_{1,0}^{2}} \frac{\partial f_{1}}{\partial b_{0,0}}=2 C_{2} \frac{b_{1,0}^{3} b_{0,1}-a_{1,0} a_{0,1}^{3}}{a_{0,0} a_{0,1} b_{1,0} b_{1,1}},
$$

therefore

$$
f_{1}=C_{2} \frac{a_{1,0}^{3} b_{0,0}^{2}}{a_{0,0}^{2} b_{1,0}^{2}}+C_{8} \frac{a_{1,0}^{2} b_{0,0}}{a_{0,0} b_{1,0}}+f_{2}\left(a_{0,0}, a_{1,0}, b_{1,0}\right) .
$$


Substitute in (3.14) the expression found instead of $f_{1}$ and differentiate with respect to $a_{2,0}$ to get

$$
D_{m}\left(\frac{\partial f_{2}}{\partial a_{1,0}}\right)-\frac{\partial f_{2}}{\partial a_{1,0}}=2\left(C_{1}-C_{8}\right) \frac{a_{1,0} a_{0,1}^{3}-b_{1,0}^{3} b_{0,1}}{a_{0,0} a_{0,1} b_{1,0} b_{1,1}}
$$

therefore

$$
C_{8}=C_{1}, \quad f_{2}=C_{9} a_{1,0}+f_{3}\left(a_{0,0}, b_{1,0}\right) .
$$

For the functions $f_{3}, g_{3}$ we have a system

$$
\begin{aligned}
& \frac{a_{1,0}}{b_{2,0}^{2}} D_{n} D_{m} f_{3}-\frac{a_{1,1}}{b_{2,0}^{2}} D_{n} f_{3}-\frac{a_{1,0} a_{0,1}}{a_{1,1} b_{2,0}^{2}} D_{m} f_{3}+\frac{a_{1,1} a_{0,0}}{a_{1,0} b_{1,0}^{2}} f_{3}-\frac{2}{b_{2,0}} D_{n} g_{3}=2\left(C_{9}-C_{3}\right), \\
& \frac{b_{1,0}}{a_{1,1}^{2}} D_{n} D_{m} g_{3}+\frac{b_{1,1}}{a_{1,1}^{2}} D_{n} g_{3}-\frac{b_{1,0} b_{0,1}}{b_{1,1} a_{0,1}^{2}} D_{m} g_{3}-\frac{b_{1,1} b_{0,0}}{b_{1,0} a_{0,1}^{2}} g_{3}+\frac{2}{a_{1,1}} D_{m} f_{3}=2\left(C_{9}-C_{3}\right) .
\end{aligned}
$$

We see that (3.16) is satisfied identically if one chooses

$$
C_{9}=C_{3}, \quad f_{3}=0, \quad g_{3}=0 .
$$

Thus we find the final form of the symmetry searched

$$
\begin{aligned}
\frac{\partial_{t} a_{1,0}}{a_{1,0}}-\frac{\partial_{t} a_{0,0}}{a_{0,0}}= & C_{1}\left(\frac{a_{0,0} b_{2,0}}{a_{1,0} b_{1,0}}+\frac{a_{1,0} b_{0,0}}{a_{0,0} b_{1,0}}\right) \\
& +C_{2}\left(\frac{a_{1,0} a_{-1,0}}{a_{0,0}^{2}}+\frac{2 b_{0,0} b_{2,0}}{b_{1,0}^{2}}+\frac{a_{1,0}^{2} b_{0,0}^{2}}{a_{0,0}^{2} b_{1,0}^{2}}+\frac{a_{0,0} a_{2,0}}{a_{1,0}^{2}}+\frac{a_{0,0}^{2} b_{2,0}^{2}}{a_{1,0}^{2} b_{1,0}^{2}}\right)+C_{3}, \\
\frac{\partial_{t} b_{1,0}}{b_{1,0}}-\frac{\partial_{t} b_{0,0}}{b_{0,0}}= & C_{1}\left(\frac{a_{-1,0} b_{1,0}}{a_{0,0} b_{0,0}}+\frac{a_{1,0} b_{0,0}}{a_{0,0} b_{1,0}}\right) \\
& +C_{2}\left(\frac{2 a_{1,0} a_{-1,0}}{a_{0,0}^{2}}+\frac{b_{0,0} b_{2,0}}{b_{1,0}^{2}}+\frac{a_{1,0}^{2} b_{0,0}^{2}}{a_{0,0}^{2} b_{1,0}^{2}}+\frac{b_{-1,0} b_{1,0}}{b_{0,0}^{2}}+\frac{a_{-1,0} b_{1,0}^{2}}{a_{0,0}^{2} b_{0,0}^{2}}\right)+C_{3} .
\end{aligned}
$$

Here $C_{1}, C_{2}, C_{3}$ are arbitrary constants. From (3.9) one can find the function $\widetilde{G}$, then (1.10) looks like

$$
\begin{aligned}
\frac{\partial_{t} a_{0,1}}{a_{0,1}}= & \frac{\partial_{t} b_{0,0}}{b_{0,0}}-\frac{\partial_{t} a_{0,0}}{a_{0,0}}+C_{1} \frac{b_{1,0}}{a_{0,0}}\left(\frac{2 a_{-1,0}}{b_{0,0}}-\frac{b_{0,0}}{a_{0,1}}\right) \\
& +C_{2}\left(\frac{2 b_{1,0} b_{-1,0}}{b_{0,0}^{2}}+\frac{2 b_{1,0}^{2} a_{-1,0}^{2}}{a_{0,0}^{2} b_{0,0}^{2}}+\frac{2 a_{1,0} a_{-1,0}}{a_{0,0}^{2}}-\frac{a_{-1,0} b_{1,0}^{2}}{a_{0,0}^{2} a_{0,1}}-\frac{b_{0,0}^{2} a_{1,0}}{a_{0,0}^{2} a_{0,1}}\right)+C_{3}, \\
\frac{\partial_{t} b_{0,1}}{b_{0,1}}= & \frac{2 \partial_{t} a_{0,0}}{a_{0,0}}-\frac{3 \partial_{t} b_{0,0}}{b_{0,0}}+C_{2}\left(\frac{2 b_{1,0} a_{-1,0}}{a_{0,0} b_{0,0}}+\frac{b_{0,0} a_{0,1}}{a_{0,0} b_{0,1}}-\frac{a_{-1,0} a_{0,1}^{2}}{a_{0,0} b_{0,0} b_{0,1}}\right) \\
& +C_{2}\left(\frac{2 a_{1,0} a_{-1,0}}{a_{0,0}^{2}}+\frac{2 b_{1,0} b_{-1,0}}{b_{0,0}^{2}}+\frac{2 a_{0,1} b_{1,0} a_{-1,0}}{a_{0,0}^{2} b_{0,1}}+\frac{2 b_{1,0}^{2} a_{-1,0}^{2}}{a_{0,0}^{2} b_{0,0}^{2}}-\frac{b_{0,0}^{2} b_{1,0}}{a_{0,0}^{2} b_{0,1}}\right. \\
& \left.-\frac{a_{0,1}^{2} b_{-1,0}}{b_{0,0}^{2} b_{0,1}}-\frac{a_{0,1}^{2} a_{-1,0}^{2} b_{1,0}}{a_{0,0}^{2} b_{0,0}^{2} b_{0,1}}\right)+C_{3} .
\end{aligned}
$$

Thus we have proved the following

Theorem 3.1. Equations (3.17) and (3.18) define a hyperbolic type symmetry for equation (3.3). 
Remark 3.2. By applying the replacement $n \leftrightarrow-m$ to the equations (3.17) and (3.18) one can obtain the second hyperbolic type symmetry for equation (3.3).

By analogy with the continuous case we have a differential constraint completely consistent with system (3.3), which is obtained from the last system by applying the operator $\left(D_{n}-1\right)^{-1}$ to the difference of the two equations

$$
\frac{\partial_{t} b_{0,0}}{b_{0,0}}-\frac{\partial_{t} a_{0,0}}{a_{0,0}}=C_{1} \frac{a_{-1,0} b_{1,0}}{a_{0,0} b_{0,0}}+C_{2}\left(\frac{a_{-1,0} a_{1,0}}{a_{0,0}^{2}}+\frac{b_{-1,0} b_{1,0}}{b_{0,0}^{2}}+\frac{a_{-1,0}^{2} b_{1,0}^{2}}{a_{0,0}^{2} b_{0,0}^{2}}\right) .
$$

Under the Cole-Hopf type transformation

$$
\hat{a}_{0,0}=\frac{a_{1,0}}{a_{0,0}}, \quad \hat{b}_{0,0}=\frac{b_{1,0}}{b_{0,0}}
$$

system (3.3) converts to the following one

$$
\begin{aligned}
& \hat{a}_{1,1} \hat{a}_{0,0} \hat{a}_{0,1}-\hat{a}_{1,0} \hat{a}_{0,1} \hat{a}_{0,0}=\hat{b}_{1,0}^{2}\left(\hat{a}_{0,0}-\hat{a}_{0,1}\right), \\
& \hat{b}_{1,1} \hat{b}_{0,0} \hat{b}_{0,1}-\hat{b}_{1,0} \hat{b}_{0,1} \hat{b}_{0,0}=\hat{a}_{0,1}^{2}\left(\hat{b}_{0,1}-\hat{b}_{0,0}\right) .
\end{aligned}
$$

This transformation brings our hyperbolic type symmetry to generalized symmetry of usual form for the new system (3.19)

$$
\begin{aligned}
& \partial_{t} \hat{a}_{0,0}=C_{1}\left(\hat{b}_{1,0}+\frac{\hat{a}_{0,0}^{2}}{\hat{b}_{0,0}}\right)+C_{2}\left(\frac{\hat{a}_{0,0}^{2}}{\hat{a}_{-1,0}}+\frac{2 \hat{b}_{1,0} \hat{a}_{0,0}}{\hat{b}_{0,0}}+\frac{\hat{a}_{0,0}^{3}}{\hat{b}_{0,0}^{2}}+\hat{a}_{1,0}+\frac{\hat{b}_{1,0}^{2}}{\hat{a}_{0,0}}\right)+C_{3} \hat{a}_{0,0}, \\
& \partial_{t} \hat{b}_{0,0}=C_{1}\left(\frac{\hat{b}_{0,0}^{2}}{\hat{a}_{-1,0}}+\hat{a}_{0,0}\right)+C_{2}\left(\frac{2 \hat{a}_{0,0} \hat{b}_{0,0}}{\hat{a}_{-1,0}}+\hat{b}_{1,0}+\frac{\hat{a}_{0,0}^{2}}{\hat{b}_{0,0}}+\frac{\hat{b}_{0,0}^{2}}{\hat{b}_{-1,0}}+\frac{\hat{b}_{0,0}^{3}}{\hat{a}_{-1,0}^{2}}\right)+C_{3} \hat{b}_{0,0} .
\end{aligned}
$$

\subsection{Hyperbolic type symmetries for the system $A_{2}^{(2)}$}

The Cartan matrix of $A_{2}^{(2)}$ is

$$
A=\left(\begin{array}{cc}
2 & -1 \\
-4 & 2
\end{array}\right)
$$

The discrete system for $A_{2}^{(2)}$ looks as

$$
\Delta\left(u^{1}\right)=e^{u_{0,1}^{1}+u_{1,0}^{1}-u_{1,0}^{2}}, \quad \Delta\left(u^{2}\right)=e^{-4 u_{0,1}^{1}+u_{0,1}^{2}+u_{1,0}^{2}} .
$$

First part of its hyperbolic type symmetry (function $G$ ) is

$$
\begin{aligned}
\left(u_{1,0}^{1}-u_{0,0}^{1}\right)_{t}= & C_{1}\left(e^{2 u_{1,0}^{1}-u_{0,0}^{1}-u_{2,0}^{1}}+e^{2 u_{1,0}^{1}-2 u_{0,0}^{1}+u_{1,0}^{2}-u_{2,0}^{2}}+e^{2 u_{0,0}^{1}-2 u_{1,0}^{1}+u_{1,0}^{2}-u_{0,0}^{2}}\right. \\
& \left.+e^{2 u_{0,0}^{1}-u_{1,0}^{1}-u_{-1,0}^{1}}\right)+C_{2}\left(3 e^{4 u_{1,0}^{1}-2 u_{0,0}^{1}-2 u_{2,0}^{1}}+e^{u_{1,0}^{1}-u_{0,0}^{1}+u_{2,0}^{1}-u_{3,0}^{1}}\right. \\
& +3 e^{-u_{2,0}^{1}+u_{1,0}^{2}+u_{0,0}^{1}-u_{0,0}^{2}}+3 e^{4 u_{1,0}^{1}-u_{2,0}^{1}-3 u_{0,0}^{1}+u_{1,0}^{2}-u_{2,0}^{2}}+2 e^{u_{1,0}^{1}-u_{2,0}^{1}+u_{0,0}^{1}-u_{-1,0}^{1}} \\
& +e^{4 u_{1,0}^{1}-4 u_{0,0}^{1}+2 u_{1,0}^{2}-2 u_{2,0}^{2}}+2 e^{u_{2,0}^{2}-2 u_{1,0}^{2}-u_{0,0}^{2}}+3 e^{u_{1,0}^{1}-u_{2,0}^{2}+u_{1,0}^{2}-u_{-1,0}^{1}} \\
& +e^{-u_{1,0}^{1}+u_{-1,0}^{1}+u_{0,0}^{1}-u_{-2,0}^{1}}+e^{-4 u_{1,0}^{1}+2 u_{1,0}^{2}-2 u_{0,0}^{2}+4 u_{0,0}^{1}} \\
& +e^{4 u_{1,0}^{1}-u_{0,0}^{1}+u_{2,0}^{2}-3 u_{2,0}^{1}-u_{1,0}^{2}}+e^{-u_{1,0}^{1}+u_{-1,0}^{1}+u_{0,0}^{2}-u_{-1,0}^{2}} \\
& +3 e^{-2 u_{1,0}^{1}-2 u_{-1,0}^{1}+4 u_{0,0}^{1}}+e^{-u_{0,0}^{1}+u_{2,0}^{2}+u_{2,0}^{1}-u_{3,0}^{2}}
\end{aligned}
$$




$$
\begin{aligned}
& \left.+3 e^{3 u_{1,0}^{1}+u_{1,0}^{2}-u_{-1,0}^{1}-u_{0,0}^{2}+4 u_{0,0}^{1}}+e^{-u_{1,0}^{1}-u_{1,0}^{2}-3 u_{-1,0}^{1}+u_{0,0}^{2}+4 u_{0,0}^{1}}\right)+C_{3}, \\
& \left(u_{1,0}^{2}-u_{0,0}^{2}\right)_{t}=2 C_{1}\left(e^{-2 u_{1,0}^{1}+u_{1,0}^{2}-u_{0,0}^{2}+2 u_{0,0}^{1}}+2 e^{-u_{1,0}^{1}-u_{-1,0}^{1}+2 u_{0,0}^{1}}+e^{-u_{1,0}^{2}+2 u_{0,0}^{1}+u_{0,0}^{2}-2 u_{-1,0}^{1}}\right) \\
& +2 C_{2}\left(2 e^{u_{1,0}^{1}-u_{2,0}^{1}+u_{0,0}^{1}-u_{-1,0}^{1}}+2 e^{-u_{2,0}^{1}+u_{1,0}^{2}+u_{0,0}^{1}-u_{0,0}^{2}}\right. \\
& +4 e^{-3 u_{1,0}^{1}+u_{1,0}^{2}-u_{-1,0}^{1}-u_{0,0}^{2}+4 u_{0,0}^{1}}+e^{-4 u_{1,0}^{1}+2 u_{1,0}^{2}-2 u_{0,0}^{2}+4 u_{0,0}^{1}} \\
& +2 e^{u_{1,0}^{1}-u_{2,0}^{2}+u_{1,0}^{2}-u_{-1,0}^{1}}+e^{-u_{2,0}^{2}+2 u_{1,0}^{2}-u_{0,0}^{2}}+2 e^{-u_{1,0}^{1}+u_{-1,0}^{1}+u_{0,0}^{1}-u_{-2,0}^{1}} \\
& +2 e^{-u_{1,0}^{1}+u_{-1,0}^{1}+u_{0,0}^{2}-u_{-1,0}^{2}}+4 e^{-u_{1,0}^{1}-u_{1,0}^{2}-3 u_{-1,0}^{1}+u_{0,0}^{2}+4 u_{0,0}^{1}} \\
& +2 e^{-u_{1,0}^{2}+u_{0,0}^{1}+u_{0,0}^{2}-u_{-2,0}^{1}}+e^{-u_{1,0}^{2}+2 u_{0,0}^{2}-u_{-1,0}^{2}}+6 e^{-2 u_{1,0}^{1}-2 u_{-1,0}^{1}+4 u_{0,0}^{1}} \\
& \left.+e^{-2 u_{1,0}^{2}+4 u_{0,0}^{1}+2 u_{0,0}^{2}-4 u_{-1,0}^{1}}\right)+2 C_{3} .
\end{aligned}
$$

Here $C_{1}, C_{2}, C_{3}$ are arbitrary constants. Second part of hyperbolic type symmetry (function $\widetilde{G}$ ) can be evaluated automatically from the compatibility conditions.

And for combination we have a local constraint

$$
\begin{aligned}
\left(2 u_{0,0}^{1}-u_{0,0}^{2}\right)_{t}= & 2 C_{1}\left(e^{2 u_{0,0}^{1}-u_{1,0}^{1}-u_{-1,0}^{1}}+e^{2 u_{0,0}^{1}-2 u_{-1,0}^{1}+u_{0,0}^{2}-u_{1,0}^{2}}\right) \\
& +2 C_{2}\left(e^{-u_{-1,0}^{1}-u_{2,0}^{1}+u_{0,0}^{1}+u_{1,0}^{1}}+e^{u_{1,0}^{1}+u_{1,0}^{2}-u_{-1,0}^{1}-u_{2,0}^{2}}\right. \\
& +e^{-3 u_{1,0}^{1}+u_{1,0}^{2}+4 u_{0,0}^{1}-u_{-1,0}^{1}-u_{0,0}^{2}}+3 e^{-2 u_{1,0}^{1}+4 u_{0,0}^{1}-2 u_{-1,0}^{1}} \\
& +3 e^{-u_{1,0}^{1}-u_{1,0}^{2}+4 u_{0,0}^{1}-3 u_{-1,0}^{1}+u_{0,0}^{2}}+e^{-u_{1,0}^{1}+u_{-1,0}^{1}+u_{0,0}^{2}-u_{-1,0}^{2}} \\
& +e^{-u_{1,0}^{1}+u_{0,0}^{1}+u_{-1,0}^{1}-u_{-2,0}^{1}}+e^{-2 u_{1,0}^{2}+4 u_{0,0}^{1}-4 u_{-1,0}^{1}+2 u_{0,0}^{2}} \\
& \left.+e^{-u_{1,0}^{2}+2 u_{0,0}^{2}-u_{-1,0}^{2}}+2 e^{-u_{1,0}^{2}+u_{0,0}^{1}+u_{0,0}^{2}-u_{-2,0}^{1}}\right)
\end{aligned}
$$

\section{Characteristic $m$-algebra for the case $A_{2}$}

Let us describe briefly the properties of the characteristic Lie algebras of the system

$$
a_{0,0} a_{1,1}=a_{1,0} a_{0,1}+b_{1,0}, \quad b_{0,0} b_{1,1}=b_{1,0} b_{0,1}+a_{0,1} .
$$

Recall that system (4.1) corresponds to the simple Lie algebra $A_{2}$. First we concentrate on the notion of the characteristic $m$-algebra for the system (4.1). Lie algebra on the other destination is studied similarly. Recall that according to the definition an $m$-integral $F\left(a_{0,0}, b_{0,0}, a_{1,0}, b_{1,0}\right.$, $\left.a_{-1,0}, b_{-1,0}, \ldots\right)$ should satisfy the equation $D_{m} F=F$. In the coordinate representation this condition reads

$$
F\left(a_{0,1}, b_{0,1}, a_{1,1}, b_{1,1}, a_{-1,1}, b_{-1,1}, \ldots\right)=F\left(a_{0,0}, b_{0,0}, a_{1,0}, b_{1,0}, a_{-1,0}, b_{-1,0}, \ldots\right) .
$$

Evidently the right hand side in (4.2) does not depend on the variables $a_{0,1}$ and $b_{0,1}$ hence the conditions hold $\frac{\partial}{\partial a_{0,1}} D_{m}^{-1} F=0, \frac{\partial}{\partial b_{0,1}} D_{m}^{-1} F=0$ as well as $Y_{1} F=0, Y_{2} F=0$ where $Y_{1}:=D_{m}^{-1} \frac{\partial}{\partial a_{0,1}} D_{m}, Y_{2}:=D_{m}^{-1} \frac{\partial}{\partial b_{0,1}} D_{m}$. Denote through $L_{m}$ the Lie algebra generated by the operators $X_{1}=\frac{\partial}{\partial a_{0,-1}}, X_{2}=\frac{\partial}{\partial b_{0,-1}}, Y_{1}, Y_{2}$. Algebra $L_{m}$ is called characteristic $m$-algebra. Obviously, operators $X_{1}, X_{2}$ are the first-order linear differential operators, or vector fields. The operators $Y_{1}, Y_{2}$ can also be rewritten as vector fields of the form

$$
Y_{1}=\frac{\partial}{\partial a_{0,0}}+\left(\frac{a_{1,0}}{a_{0,0}}-\frac{b_{1,0} b_{0,-1}}{a_{0,0} b_{0,0} a_{0,-1}}\right) \frac{\partial}{\partial a_{1,0}}+\left(\frac{a_{-1,0}}{a_{0,0}}+\frac{b_{0,-1}}{a_{0,0} a_{0,-1}}\right) \frac{\partial}{\partial a_{-1,0}}+\cdots
$$




$$
\begin{aligned}
& +\frac{1}{b_{0,-1}} \frac{\partial}{\partial b_{1,0}}-\left(\frac{a_{-1,0}}{a_{0,0} b_{0,-1}}+\frac{1}{a_{0,0} a_{0,-1}}\right) \frac{\partial}{\partial b_{-1,0}}+\cdots, \\
Y_{2}= & \frac{\partial}{\partial b_{0,0}}+\left(\frac{b_{1,0}}{b_{0,0}}-\frac{a_{0,0}}{b_{0,0} b_{0,-1}}\right) \frac{\partial}{\partial b_{1,0}}+\left(\frac{b_{-1,0}}{b_{0,0}}+\frac{a_{-1,0}}{b_{0,0} b_{0,-1}}\right) \frac{\partial}{\partial b_{-1,0}}+\cdots .
\end{aligned}
$$

We use the following lemma to show that $m$-algebra is of finite dimension.

Lemma 4.1. Suppose that the vector field

$$
K=\sum_{k=1}^{\infty}\left(\alpha_{k} \frac{\partial}{\partial a_{k}}+\alpha_{-k} \frac{\partial}{\partial a_{-k}}\right)+\sum_{k=1}^{\infty}\left(\beta_{k} \frac{\partial}{\partial b_{k}}+\beta_{-k} \frac{\partial}{\partial b_{-k}}\right)
$$

satisfies the equality $D_{n} K D_{n}^{-1}=h K$, where $h$ is a function depending on shifts of variables a and $b$, then $K=0$.

Lemma can be proved by applying both sides of the equation $D_{n} K D_{n}^{-1}=h K$ to the dynamical variables $a_{k}, b_{k}$.

One can easily check that

$$
\begin{aligned}
& D_{n} X_{1} D_{n}^{-1}=\frac{a_{0,0}}{a_{1,0}} X_{1}, \quad D_{n} X_{2} D_{n}^{-1}=\frac{1}{a_{1,0}} X_{1}+\frac{b_{0,0}}{b_{1,0}} X_{2}, \\
& D_{n} Y_{1} D_{n}^{-1}=\frac{a_{0,-1}}{a_{1,-1}} Y_{1}-\frac{a_{0,-1}}{a_{1,-1} b_{1,-1}} Y_{2}, \quad D_{n} Y_{2} D_{n}^{-1}=\frac{b_{0,-1}}{b_{1,-1}} Y_{2} .
\end{aligned}
$$

Put $\tilde{X}_{1}=a_{0,0} X_{1}, \tilde{X}_{2}=b_{0,0} X_{2}, \tilde{Y}_{1}=a_{0,-1} Y_{1}, \tilde{Y}_{2}=b_{0,-1} Y_{2}$, then

$$
\begin{aligned}
& D_{n} \tilde{X}_{1} D_{n}^{-1}=\tilde{X}_{1}, \quad D_{n} \tilde{X}_{2} D_{n}^{-1}=\frac{b_{1,0}}{a_{0,0} a_{1,0}} \tilde{X}_{1}+\tilde{X}_{2}, \\
& D_{n} \tilde{Y}_{1} D_{n}^{-1}=\tilde{Y}_{1}-\frac{a_{0,-1}}{b_{0,-1} b_{1,-1}} \tilde{Y}_{2}, \quad D_{n} \tilde{Y}_{2} D_{n}^{-1}=\tilde{Y}_{2} .
\end{aligned}
$$

Taking commutators of the vector fields $\tilde{X}_{1}, \tilde{X}_{2}, \tilde{Y}_{1}, \tilde{Y}_{2}$ we get vector fields

$$
P_{1}=\left[X_{1}, Y_{1}\right], \quad P_{2}=\left[X_{2}, Y_{1}\right], \quad P_{3}=\left[X_{2}, Y_{2}\right] .
$$

\section{Lemma 4.2.}

$$
\begin{aligned}
D_{n} P_{1} D_{n}^{-1}= & P_{1}-\frac{a_{0,0}}{b_{0,-1} b_{1,-1}} \tilde{Y}_{2}, \\
D_{n} P_{2} D_{n}^{-1}= & P_{2}+\frac{b_{1,0}}{a_{0,0} a_{1,0}} P_{1}-\frac{a_{0,-1}}{b_{0,-1} b_{1,-1}} P_{3} \\
& +\left(\frac{a_{0,-1} b_{1,0}}{a_{0,0}^{2} a_{1,0}}+\frac{a_{1,-1} b_{1,0}}{a_{0,0} a_{1,0}^{2}}\right) \tilde{X}_{1}+\left(\frac{a_{0,-1} b_{0,0}}{b_{0,-1}^{2} b_{1,-1}}+\frac{a_{1,-1} a_{0,0} b_{1,0}}{a_{1,0} b_{0,-1} b_{1,-1}^{2}}\right) \tilde{Y}_{2}, \\
D_{n} P_{3} D_{n}^{-1}= & P_{3}-\frac{b_{1,-1}}{a_{0,0} a_{1,0}} \tilde{X}_{1} .
\end{aligned}
$$

Lemma can be proved by direct calculations. It allows to derive the following table of commutators which shows that the characteristic Lie algebra $L_{m}$ is of dimension seven:

\begin{tabular}{|c|c|c|c|c|c|c|c|}
\hline & $\tilde{X}_{1}$ & $\tilde{X}_{2}$ & $\tilde{Y}_{1}$ & $\tilde{Y}_{2}$ & $P_{1}$ & $P_{2}$ & $P_{3}$ \\
\hline$\tilde{X}_{1}$ & 0 & 0 & $P_{1}$ & 0 & 0 & $R_{1}$ & 0 \\
\hline$\tilde{X}_{2}$ & 0 & 0 & $P_{2}$ & $P_{3}$ & $R_{1}$ & $R_{2}$ & $-2 \tilde{X}_{2}$ \\
\hline$\tilde{Y}_{1}$ & $-P_{1}$ & $-P_{2}$ & 0 & 0 & $2 \tilde{Y}_{1}$ & $R_{3}$ & $R_{4}$ \\
\hline$\tilde{Y}_{2}$ & 0 & $-P_{3}$ & 0 & 0 & 0 & $R_{4}$ & 0 \\
\hline$P_{1}$ & 0 & $-R_{1}$ & $-2 \tilde{Y}_{1}$ & 0 & 0 & $Q_{1}$ & $Q_{2}$ \\
\hline$P_{2}$ & $-R_{1}$ & $-R_{2}$ & $-R_{3}$ & $-R_{4}$ & $-Q_{1}$ & 0 & $Q_{3}$ \\
\hline$P_{3}$ & 0 & $2 \tilde{X}_{2}$ & $-R_{4}$ & 0 & $-Q_{2}$ & $-Q_{3}$ & 0 \\
\hline
\end{tabular}


Here the following notations are used

$$
\begin{aligned}
R_{1} & =\tilde{X}_{2}-\frac{b_{1,0}}{b_{0,-1}} P_{1}+\frac{b_{0,0}^{2}}{b_{0,-1}^{2}} \tilde{Y}_{2}, \\
R_{2} & =\frac{2 a_{0,-1} b_{0,0}^{2}}{a_{0,0} b_{0,-1}^{2}} P_{3}-\frac{2 a_{0,-1} b_{0,0}^{3}}{a_{0,0} b_{0,-1}^{3}} \tilde{Y}_{2}+\frac{2 a_{0,-1} b_{0,0}}{a_{0,0} b_{0,-1}} \tilde{X}_{2}, \\
R_{3} & =\frac{2 a_{0,-1}^{2} b_{0,0}}{a_{0,0}^{2} b_{0,-1}} P_{1}-\frac{2 a_{0,-1} b_{0,0}}{a_{0,0} b_{0,-1}} \tilde{Y}_{1}+\frac{2 a_{0,-1}^{3} b_{0,0}}{a_{0,0}^{3} b_{0,-1}} \tilde{X}_{1}, \\
R_{4} & =-\tilde{Y}_{1}-\frac{a_{0,-1}^{2}}{a_{0,0}^{2}} \tilde{X}_{1}-\frac{a_{0,-1}}{a_{0,0}} P_{1}, \\
Q_{1} & =\frac{a_{0,-1} b_{0,0}}{a_{0,0} b_{0,-1}} P_{1}-3 P_{2}-P_{3}+\frac{a_{0,-1}^{2} b_{0,0}}{a_{0,0}^{2} b_{0,-1}} \tilde{X}_{1}+\frac{b_{0,0}}{b_{0,-1}} \tilde{Y}_{1}, \\
Q_{2} & =\frac{2 b_{0,0}}{b_{0,-1}} \tilde{Y}_{2}-2 P_{3}, \\
Q_{3} & =-3 P_{2}+\frac{a_{0,-1} b_{0,0}}{a_{0,0} b_{0,-1}} P_{3}-\frac{a_{0,-1}}{a_{0,0}} \tilde{X}_{2}-\frac{a_{0,-1} b_{0,0}^{2}}{a_{0,0} b_{0,-1}^{2}} \tilde{Y}_{2} .
\end{aligned}
$$

Due to the reasonings above any $m$-integral $F\left(\ldots, a_{0,0}, b_{0,0}, a_{1,0}, b_{1,0}, a_{2,0}, b_{2,0}, \ldots\right)$ should satisfy equations

$$
\begin{array}{llll}
\tilde{X}_{1}(F)=0, & \tilde{X}_{2}(F)=0, & \tilde{Y}_{1}(F)=0, & \tilde{Y}_{2}(F)=0, \\
P_{1}(F)=0, & P_{2}(F)=0, & P_{3}(F)=0 .
\end{array}
$$

Solving system (4.3), it is enough to assume that $F$ depends on $b_{0,0}, a_{0,0}, b_{1,0}, a_{1,0}, b_{2,0}$, $a_{2,0}$ or, alternatively, $F$ depends on $a_{-1,0}, b_{0,0}, a_{0,0}, b_{1,0}, a_{1,0}, b_{2,0}$. Under such assumptions system (4.3) generates two systems of the first-order linear partial differential equations which

can be solved by Jacobi method. By solving these systems we find two independent $m$-integrals

$$
F_{(1)}=\frac{b_{0,0}}{b_{1,0}}+\frac{a_{0,0} b_{2,0}}{a_{1,0} b_{1,0}}+\frac{a_{2,0}}{a_{1,0}}, \quad F_{(2)}=\frac{a_{-1,0}}{a_{0,0}}+\frac{a_{1,0} b_{0,0}}{a_{0,0} b_{1,0}}+\frac{b_{2,0}}{b_{1,0}} .
$$

\section{Cutting off constraints for the Hirota equation and discrete Zakharov-Shabat systems}

In this section we construct Lax pairs for discrete systems corresponding to Cartan matrices of series $A_{N}, B_{N}, C_{N}, D_{N}^{(2)}$ and $A_{1}^{(1)}$. To this end we impose cutting off constrains for the Hirota equation compatible with its Lax pair.

It is well known that Hirota chain

$$
t_{0,0}^{j} t_{1,1}^{j}-t_{1,0}^{j} t_{0,1}^{j}=t_{1,0}^{j-1} t_{0,1}^{j+1}
$$

admits the Lax pair consisting of two linear discrete equations [7]

$$
\psi_{1,0}^{j}=\frac{t_{1,0}^{j+1} t_{0,0}^{j}}{t_{0,0}^{j+1} t_{1,0}^{j}} \psi_{0,0}^{j}-\psi_{0,0}^{j+1}, \quad \psi_{0,1}^{j}=\psi_{0,0}^{j}+\frac{t_{0,1}^{j+1} t_{0,0}^{j-1}}{t_{0,0}^{j} t_{0,1}^{j}} \psi_{0,0}^{j-1} .
$$

Here the lower indices mean as previously shifts of the arguments, and the upper index enumerates the field variables $t^{j}$ and eigenfunctions $\psi^{j}$. Exclude from the system of equations (5.2) all 
the eigenfunctions with the upper index different from $j$. As a result one gets a linear discrete hyperbolic equation for $\psi^{j}$

$$
\psi_{1,1}^{j}-\psi_{1,0}^{j}-\frac{t_{1,1}^{j+1} t_{0,1}^{j}}{t_{0,1}^{j+1} t_{1,1}^{j}} \psi_{0,1}^{j}+\frac{t_{0,0}^{j} t_{1,1}^{j+1}}{t_{1,0}^{j} t_{0,1}^{j+1}} \psi_{0,0}^{j}=0 .
$$

It is remarkable that by construction formulas (5.2) define Laplace transformations for the linear hyperbolic equation (5.3). Evidently, equation (5.1) is invariant under the transformation defined as $n \rightarrow 1-m, m \rightarrow 1-n$. Under this transformation the Lax pair (5.2) transforms to a Lax pair

$$
y_{-1,0}^{j}=y_{0,0}^{j}+\frac{t_{-1,0}^{j+1} t_{0,0}^{j-1}}{t_{-1,0}^{j} t_{0,0}^{j}} y_{0,0}^{j-1}, \quad y_{0,-1}^{j}=\frac{t_{0,-1}^{j+1} t_{0,0}^{j}}{t_{0,0}^{j+1} t_{0,-1}^{j}} y_{0,0}^{j}-y_{0,0}^{j+1},
$$

and equation (5.3) transforms to an equation

$$
y_{1,1}^{j}-\frac{t_{1,0}^{j} t_{0,1}^{j+1}}{t_{0,0}^{j+1} t_{1,1}^{j}} y_{1,0}^{j}-\frac{t_{1,0}^{j} t_{0,1}^{j}}{t_{0,0}^{j} t_{1,1}^{j}} y_{0,1}^{j}+\frac{t_{1,0}^{j} t_{0,1}^{j+1}}{t_{0,0}^{j+1} t_{1,1}^{j}} y_{0,0}^{j}=0 .
$$

Put $y_{0,0}^{j}=\frac{t_{-1,0}^{j+1}}{t_{0,0}^{j}} g_{0,0}^{j}$, then Lax pair (5.4) transforms to a Lax pair

$$
g_{-1,0}^{j}=\frac{t_{-1,0}^{j+1} t_{-1,0}^{j}}{t_{0,0}^{j} t_{-2,0}^{j+1}}\left(g_{0,0}^{j}+g_{0,0}^{j-1}\right), \quad g_{0,-1}^{j}=\frac{t_{0,-1}^{j+1} t_{-1,0}^{j+1}}{t_{0,0}^{j+1} t_{-1,-1}^{j+1}} g_{0,0}^{j}-\frac{t_{0,-1}^{j} t_{-1,0}^{j+2}}{t_{0,0}^{j+1} t_{-1,-1}^{j+1}} g_{0,0}^{j+1},
$$

and equation (5.5) transforms to an equation

$$
g_{1,1}^{j}-g_{1,0}^{j}-\frac{t_{1,0}^{j} t_{-1,1}^{j+1}}{t_{0,1}^{j+1} t_{0,0}^{j}} g_{0,1}^{j}+\frac{t_{1,0}^{j} t_{-1,0}^{j+1}}{t_{0,0}^{j+1} t_{0,0}^{j}} g_{0,0}^{j}=0 .
$$

Equations (5.6) define Laplace transformations for the linear hyperbolic equation (5.7). Thus we have two different Lax pairs for the Hirota chain and consequently two families (5.3), (5.7) of linear discrete hyperbolic equations enumerated by $j$. Study the question when an equation from one family can be related, by a linear transformation, to an equation from the other family. To this end we evaluate the Laplace invariants of these equations.

Recall that the Laplace invariants of a discrete hyperbolic type equation of the form

$$
a_{0,0} f_{1,1}+b_{0,0} f_{1,0}+c_{0,0} f_{0,1}+d_{0,0} f_{0,0}=0
$$

are given by (see $[2,8,31,33]$ )

$$
K_{1}=\frac{b_{0,0} c_{1,0}}{a_{0,0} d_{1,0}}, \quad K_{2}=\frac{b_{0,1} c_{0,0}}{a_{0,0} d_{0,1}} .
$$

By virtue of these formulas the invariants $K_{1 \psi}, K_{2 \psi}$ and $K_{1 g}, K_{2 g}$ of equations (5.3), (5.7) are, respectively,

$$
K_{1 \psi}=\frac{t_{2,0}^{j} t_{1,1}^{j}}{t_{1,0}^{j} t_{2,1}^{j}}, \quad K_{2 \psi}=\frac{t_{1,1}^{j+1} t_{0,2}^{j+1}}{t_{0,1}^{j+1} t_{1,2}^{j+1}}, \quad K_{1 g}=\frac{t_{0,1}^{j+1} t_{1,0}^{j+1}}{t_{0,0}^{j+1} t_{1,1}^{j+1}}, \quad K_{2 g}=\frac{t_{1,0}^{j} t_{0,1}^{j}}{t_{0,0}^{j} t_{1,1}^{j}} .
$$

It is known that two linear hyperbolic type equations are related to one another by a linear transformation only if their corresponding Laplace invariants are equal. Evidently in generic 
case coincidence of the Laplace invariants generates two constraints on the field variables $t^{j}=$ $t^{j}(n, m)$. Only for some special cases it gives only one constraint. We are interested in such special cases. For instance pair of equations $K_{1 \psi}(n, m, j)=K_{1 g}(n+1, m, j-1), K_{2 \psi}(n, m, j)=$ $K_{2 g}(n+1, m, j-1)$ is equivalent to the constraint

$$
t_{1,0}^{j-1}=t_{0,1}^{j+1}
$$

which is interpreted as a cutting off boundary condition for the chain (5.1). For simplicity we put $j=0$, so the boundary condition becomes

$$
t_{1,0}^{-1}=t_{0,1}^{1} \text {. }
$$

Lemma 5.1. Hirota equation (5.1) is compatible with the reduction of the type of parity

$$
t_{m+i, k}^{-m}=t_{i, m+k}^{m}
$$

and boundary condition (5.8) is a consequence of this reduction.

Following [15] we can construct a Lax pair for the reduced chain. Under the boundary condition (5.8) we have coincidence of the invariants

$$
K_{1 \psi}(n, m, j)=K_{1 g}(n+1, m, j-1), \quad K_{2 \psi}(n, m, j)=K_{2 g}(n+1, m, j-1),
$$

and

$$
K_{1 g}(n, m, j)=K_{1 \psi}(n, m-1, j-1), \quad K_{2 g}(n, m, j)=K_{2 \psi}(n, m-1, j-1),
$$

and we can relate the eigenfunctions

$$
g_{0,1}^{1}=\lambda \psi_{0,0}^{0}, \quad g_{1,0}^{0}=\lambda \psi_{0,0}^{1} .
$$

We study the finite reductions of the chain (5.1) on a finite interval $N_{L} \leq j \leq N_{R}$. The reduction is obtained by imposing the boundary conditions at the left end-point $j=N_{L}$

$$
t_{1,0}^{N_{L}-1}=t_{0,1}^{N_{L}+1}
$$

and respectively at the right end-point $j=N_{R}$

$$
t_{0,1}^{N_{R}+1}=t_{1,0}^{N_{R}-1} .
$$

First we concentrate on the left end-point. Due to the reasonings above the eigenfunctions should satisfy the following gluing conditions

$$
\psi_{0,0}^{N_{L}-1}=\frac{1}{\lambda} g_{0,1}^{N_{L}}, \quad g_{0,0}^{N_{L}-1}=\lambda \psi_{-1,0}^{N_{L}} .
$$

These conditions allow one to close the Lax equations at the left end-point

$$
g_{-1,0}^{N_{L}}=\frac{t_{-1,0}^{N_{L}+1} t_{-1,0}^{N_{L}}}{t_{0,0}^{N_{L}} t_{-2,0}^{N_{L}+1}}\left(g_{0,0}^{N_{L}}+\lambda \psi_{-1,0}^{N_{L}}\right), \quad \psi_{0,1}^{N_{L}}=\psi_{0,0}^{N_{L}}+\frac{1}{\lambda} \frac{t_{0,1}^{N_{L}+1} t_{-1,1}^{N_{L}+1}}{t_{0,0}^{N_{L}} t_{0,1}^{N_{L}}} g_{0,1}^{N_{L}} .
$$

From (5.2), (5.6) we have

$$
g_{0,-1}^{N_{L}}=\frac{t_{0,-1}^{N_{L}+1} t_{-1,0}^{N_{L}+1}}{t_{0,0}^{N_{L}+1} t_{-1,-1}^{N_{L}+1}} g_{0,0}^{N_{L}}-\frac{t_{0,-1}^{N_{L}} t_{-1,0}^{N_{L}+2}}{t_{0,0}^{N_{L}+1} t_{-1,-1}^{N_{L}+1}} g_{0,0}^{N_{L}+1}, \quad \psi_{1,0}^{N_{L}}=\frac{t_{1,0}^{N_{L}+1} t_{0,0}^{N_{L}}}{t_{0,0}^{N_{L}+1} t_{1,0}^{N_{L}}} \psi_{0,0}^{N_{L}}-\psi_{0,0}^{N_{L}+1} .
$$


To derive similar equations at the point $N_{R}$ we use the right end-point constraint (5.10), for which we have

$$
g_{0,0}^{N_{R}}=\psi_{0,-1}^{N_{R}-1}, \quad \psi_{0,0}^{N_{R}}=g_{1,0}^{N_{R}-1} .
$$

These conditions allow one to close the Lax equations at the right end-point

$$
\begin{aligned}
& \psi_{1,0}^{N_{R}-1}=\frac{t_{0,0}^{N_{R}-1} t_{1,0}^{N_{R}}}{t_{1,0}^{N_{R}-1} t_{0,0}^{N_{R}}} \psi_{0,0}^{N_{R}-1}-g_{1,0}^{N_{R}-1}, \\
& g_{0,-1}^{N_{R}-1}=\frac{t_{0,-1}^{N_{R}} t_{-1,0}^{N_{R}}}{t_{0,0}^{N_{R}} t_{-1,-1}^{N_{R}}} g_{0,0}^{N_{R}-1}-\frac{\left(t_{0,-1}^{N_{R}-1}\right)^{2}}{t_{0,0}^{N_{R}} t_{-1,-1}^{N_{R}}} \psi_{0,-1}^{N_{R}-1} .
\end{aligned}
$$

From (5.2), (5.6) we have

$$
g_{-1,0}^{N_{R}-1}=\frac{t_{-1,0}^{N_{R}} t_{-1,0}^{N_{R}-1}}{t_{0,0}^{N_{R}-1} t_{-2,0}^{N_{R}}}\left(g_{0,0}^{N_{R}-1}+g_{0,0}^{N_{R}-2}\right), \quad \psi_{0,1}^{N_{R}-1}=\psi_{0,0}^{N_{R}-1}+\frac{t_{0,1}^{N_{R}} t_{0,0}^{N_{R}-2}}{t_{0,0}^{N_{R}-1} t_{0,1}^{N_{R}-1}} \psi_{0,0}^{N_{R}-2} .
$$

The Lax pair found above is not convenient to work with because the operators contain shifts in opposite directions. Below we show that it can be rewritten in a usual form.

Shift of equation (5.11) forward with respect to the variable $n$ brings it to the form

$$
\begin{aligned}
& g_{1,0}^{N_{L}}=\frac{t_{-1,0}^{N_{L}+1} t_{1,0}^{N_{L}}}{t_{0,0}^{N_{L}} t_{0,0}^{N_{L}+1}} g_{0,0}^{N_{L}}-\lambda \psi_{0,0}^{N_{L}}, \\
& g_{1,0}^{j}=\frac{t_{-1,0}^{j+1} t_{1,0}^{j}}{t_{0,0}^{j} t_{0,0}^{j+1}} g_{0,0}^{j}-g_{1,0}^{j-1}=\sum_{k=N_{L}}^{j}(-1)^{j-k} \frac{t_{1,0}^{k} t_{-1,0}^{k+1}}{t_{0,0}^{k} t_{0,0}^{k+1}} g_{0,0}^{k}+(-1)^{j+1} \lambda \psi_{0,0}^{N_{L}}, \\
& N_{L}+1 \leq j \leq N_{R}-1 .
\end{aligned}
$$

Shift of equation (5.12) forward with respect to the variable $m$ brings it to the form

$$
\begin{aligned}
g_{0,1}^{N_{R}-1} & =\frac{t_{0,1}^{N_{R}} t_{-1,0}^{N_{R}}}{t_{0,0}^{N_{R}} t_{-1,1}^{N_{R}}} g_{0,0}^{N_{R}-1}+\frac{\left(t_{0,0}^{N_{R}-1}\right)^{2}}{t_{0,0}^{N_{R}} t_{-1,1}^{N_{R}}} \psi_{0,0}^{N_{R}-1}, \\
g_{0,1}^{j} & =\frac{t_{-1,0}^{j+1} t_{0,1}^{j+1}}{t_{0,0}^{j+1} t_{-1,1}^{j+1}} g_{0,0}^{j}+\frac{t_{-1,0}^{j+1} t_{0,1}^{j+1}}{t_{0,0}^{j+1} t_{-1,1}^{j+1}} g_{0,1}^{j+1}= \\
& =\frac{t_{-1,0}^{j+1} t_{0,1}^{j+1}}{t_{0,0}^{j+1} t_{-1,1}^{j+1}} g_{0,0}^{j}+\sum_{k=j+1}^{N_{R}-1} \frac{t_{0,0}^{j} t_{-1,0}^{k+1} t_{0,1}^{k+1}}{t_{-1,1}^{j+1} t_{0,0}^{k} t_{0,0}^{k+1}} g_{0,0}^{k}+\frac{t_{0,0}^{j} t_{0,0}^{N_{R}-1}}{t_{0,0}^{j+1} t_{0,0}^{N_{R}}} \psi_{0,0}^{N_{R}-1}, \quad N_{L} \leq j \leq N_{R}-1 .
\end{aligned}
$$

So we have the following system of linear equations

$$
\begin{aligned}
& \psi_{1,0}^{j}=\frac{t_{1,0}^{j+1} t_{0,0}^{j}}{t_{0,0}^{j+1} t_{1,0}^{j}} \psi_{0,0}^{j}-\psi_{0,0}^{j+1}, \quad N_{L} \leq j \leq N_{R}-2, \\
& \psi_{1,0}^{N_{R}-1}=\frac{t_{0,0}^{N_{R}-1} t_{1,0}^{N_{R}}}{t_{1,0}^{N_{R}-1} t_{0,0}^{N_{R}}} \psi_{0,0}^{N_{R}-1}+\sum_{k=N_{L}}^{N_{R}-1}(-1)^{N_{R}-k} \frac{t_{1,0}^{k} t_{-1,0}^{k+1}}{t_{0,0}^{k} t_{0,0}^{k+1}} g_{0,0}^{k}+(-1)^{N_{R}-1} \lambda \psi_{0,0}^{N_{L}}, \\
& \psi_{0,1}^{N_{L}}=\psi_{0,0}^{N_{L}}+\frac{1}{\lambda} \frac{t_{0,1}^{N_{L}+1} t_{0,0}^{N_{R}-1}}{t_{0,1}^{N_{L}} t_{0,0}^{N_{R}}} \psi_{0,0}^{N_{R}-1}+\sum_{j=N_{L}}^{N_{R}-1} \frac{1}{\lambda} \frac{t_{0,1}^{N_{L}+1} t_{0,1}^{k+1} t_{-1,0}^{k+1}}{t_{0,1}^{N_{L}} t_{0,0}^{k} t_{0,0}^{k+1}} g_{0,0}^{k},
\end{aligned}
$$




$$
\begin{aligned}
& \psi_{0,1}^{j}=\psi_{0,0}^{j}+\frac{t_{0,1}^{j} t_{0,0}^{j-2}}{t_{0,0}^{j-1} t_{0,1}^{j-1}} \psi_{0,0}^{j-1}, \quad N_{L}+1 \leq j \leq N_{R}-1, \\
& g_{1,0}^{j}=\sum_{k=N_{L}}^{j}(-1)^{j-k} \frac{t_{1,0}^{k} t_{-1,0}^{k+1}}{t_{0,0}^{k} t_{0,0}^{k+1}} g_{0,0}^{k}+(-1)^{j+1} \lambda \psi_{0,0}^{N_{L}}, \quad N_{L} \leq j \leq N_{R}-1, \\
& g_{0,1}^{j}=\frac{t_{-1,0}^{j+1} t_{0,1}^{j+1}}{t_{0,0}^{j+1} t_{-1,1}^{j+1}} g_{0,0}^{j}+\sum_{k=j+1}^{N_{R}-1} \frac{t_{0,0}^{j} t_{-1,0}^{k+1} t_{0,1}^{k+1}}{t_{-1,1}^{j+1} t_{0,0}^{k} t_{0,0}^{k+1}} g_{0,0}^{k}+\frac{t_{0,0}^{j} t_{0,0}^{N_{R}-1}}{t_{0,0}^{j+1} t_{0,0}^{N_{R}}} \psi_{0,0}^{N_{R}-1}, \\
& N_{L} \leq j \leq N_{R}-1 .
\end{aligned}
$$

In the next subsection we gather these equations to a matrix form.

\subsection{Lax pair for systems corresponding to the algebras $D_{N}^{(2)}, A_{1}^{(1)}$}

Imposing of non-degenerate boundary conditions (5.9), (5.10) at both end-points $N_{L}=0$ and $N_{R}=N$ leads to the system corresponding to the matrix $D_{N+1}^{(2)}, N \geq 2$

$$
\begin{aligned}
& t_{0,0}^{0} t_{1,1}^{0}-t_{1,0}^{0} t_{0,1}^{0}=\left(t_{0,1}^{1}\right)^{2}, \quad t_{0,0}^{j} t_{1,1}^{j}-t_{1,0}^{j} t_{0,1}^{j}=t_{1,0}^{j-1} t_{0,1}^{j+1}, \quad 1 \leq j \leq N-1, \\
& t_{0,0}^{N} t_{1,1}^{N}-t_{1,0}^{N} t_{0,1}^{N}=\left(t_{1,0}^{N-1}\right)^{2} .
\end{aligned}
$$

Write the set of equations (5.13)-(5.18) in the form of a Lax pair for system (5.19). Denote the eigenvector as follow $P=\left(\psi^{0}, \psi^{1}, \ldots, \psi^{N-1}, g^{0}, g^{1}, \ldots, g^{N-1}\right)^{T}$. Introduce $2 N \times 2 N$ matrices

$$
\begin{aligned}
& A=\left(\begin{array}{cccccccc}
\frac{t_{1,0}^{1} t_{0,0}^{0}}{t_{0,0}^{1} t_{1,0}^{0}} & -1 & 0 & \ldots & 0 & 0 & \ldots & 0 \\
0 & \frac{t_{1,0}^{2} t_{0,0}^{1}}{t_{0,0}^{2} t_{1,0}^{1}}-1 & \ldots & 0 & 0 & \ldots & 0 \\
(-1)^{N-1} \lambda & 0 & \ldots & \frac{t_{1,0}^{N} t_{0,0}^{N-1}}{t_{0,0}^{N} t_{1,0}^{N-1}} & (-1)^{N} \frac{t_{1,0}^{0} t_{-1,0}^{1}}{t_{0,0}^{0} t_{0,0}^{1}} & (-1)^{N-1} \frac{t_{1,0}^{1} t_{-1,0}^{2}}{t_{0,0}^{1} t_{0,0}^{2}} & \ldots & -\frac{t_{1,0}^{N-1} t_{-1,0}^{N}}{t_{0,0}^{N-1} t_{0,0}^{N}} \\
-\lambda & 0 & \ldots & 0 & \frac{t_{1,0}^{0} t_{-1,0}^{1}}{t_{0,0}^{0} t_{0,0}^{1}} & 0 & \ldots & 0 \\
\lambda & 0 & \ldots & 0 & -\frac{t_{1,0}^{0} t_{-1,0}^{1}}{t_{0,0}^{0} t_{0,0}^{1}} & \frac{t_{1,0}^{1} t_{-1,0}^{2}}{t_{0,0}^{1} t_{0,0}^{2}} & \ldots & 0 \\
(-1)^{i+1} \lambda & 0 & \ldots & 0 & \ldots & (-1)^{i-j} \frac{t_{1,0}^{j} t_{-1,0}^{j+1}}{t_{0,0}^{j} t_{0,0}^{j+1}} & \ldots & 0 \\
(-1)^{N} \lambda & 0 & \ldots & 0 & (-1)^{N-1} \frac{t_{1,0}^{0} t_{-1,0}^{1}}{t_{0,0}^{0} t_{0,0}^{1}} & (-1)^{N} \frac{t_{1,0}^{1} t_{-1,0}^{2}}{t_{0,0}^{1} t_{0,0}^{2}} & \ldots & \frac{t_{1,0}^{N-1} t_{-1,0}^{N}}{t_{0,0}^{N-1} t_{0,0}^{N}}
\end{array}\right),
\end{aligned}
$$

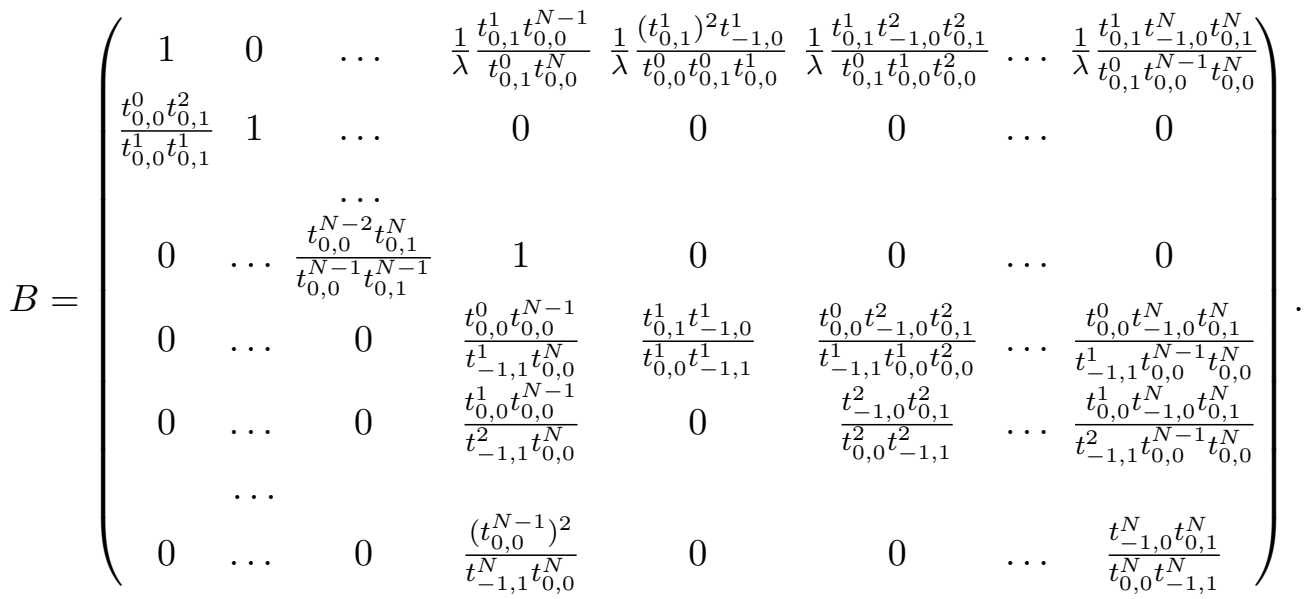


It is straightforward to check that the compatibility condition of the equations

$$
P_{1,0}=A P, \quad P_{0,1}=B P
$$

is equivalent the system (5.19).

Example 5.2. Consider a particular case when $N_{L}=0$ and $N_{R}=1$

$$
t_{0,0}^{0} t_{1,1}^{0}-t_{1,0}^{0} t_{0,1}^{0}=\left(t_{0,1}^{1}\right)^{2}, \quad t_{0,0}^{1} t_{1,1}^{1}-t_{1,0}^{1} t_{0,1}^{1}=\left(t_{1,0}^{0}\right)^{2} .
$$

The system corresponds to algebra $A_{1}^{(1)}$. Its Lax pair is given by (5.20) with the matrices

$$
A=\left(\begin{array}{cc}
\frac{t_{1,0}^{1} t_{0,0}^{0}}{t_{0,0}^{1} t_{1,0}^{0}}+\lambda & -\frac{t_{1,0}^{0} t_{-1,0}^{1}}{t_{0,0}^{0} t_{0,0}^{1}} \\
-\lambda & \frac{t_{1,0}^{0} t_{-1,0}^{1}}{t_{0,0}^{0} t_{0,0}^{1}}
\end{array}\right), \quad B=\left(\begin{array}{cc}
1+\frac{1}{\lambda} \frac{t_{0,1}^{1} t_{0,0}^{0}}{t_{0,1}^{0} t_{0,0}^{1}} & \frac{1}{\lambda} \frac{\left(t_{0,1}^{1}\right)^{2} t_{-1,0}^{1}}{t_{0,0}^{0} t_{0,1}^{0} t_{0,0}^{1}} \\
\frac{\left(t_{0,0}^{0}\right)^{2}}{t_{0,0}^{1} t_{-1,1}^{1}} & \frac{t_{0,1}^{1} t_{-1,0}^{1}}{t_{0,0}^{1} t_{-1,1}^{1}}
\end{array}\right) .
$$

Example 5.3. If one imposes condition (5.9) for $N_{L}=0$ and (5.10) for $N_{R}=2$, then

$$
t_{0,0}^{0} t_{1,1}^{0}-t_{1,0}^{0} t_{0,1}^{0}=\left(t_{0,1}^{1}\right)^{2}, \quad t_{0,0}^{1} t_{1,1}^{1}-t_{1,0}^{1} t_{0,1}^{1}=t_{1,0}^{0} t_{0,1}^{2}, \quad t_{0,0}^{2} t_{1,1}^{2}-t_{1,0}^{2} t_{0,1}^{2}=\left(t_{1,0}^{1}\right)^{2} .
$$

The Lax pair is of the form (5.20) where $A$ and $B$ are $4 \times 4$ matrices

$$
\begin{aligned}
A & =\left(\begin{array}{cccc}
\frac{t_{1,0}^{1} t_{0,0}^{0}}{t_{0,0}^{1} t_{1,0}^{0}} & -1 & 0 & 0 \\
-\lambda & \frac{t_{1,0}^{2} t_{0,0}^{1}}{t_{0,0}^{2} t_{1,0}^{1}} & \frac{t_{1,0}^{0} t_{-1,0}^{1}}{t_{0,0}^{0} t_{0,0}^{1}} & -\frac{t_{1,0}^{1} t_{-1,0}^{2}}{t_{0,0}^{1} t_{0,0}^{2}} \\
-\lambda & 0 & \frac{t_{1,0}^{0} t_{-1,0}^{1}}{t_{0,0}^{0} t_{0,0}^{1}} & 0 \\
\lambda & 0 & -\frac{t_{1,0}^{0} t_{-1,0}^{1}}{t_{0,0}^{0} t_{0,0}^{1}} & \frac{t_{1,0}^{1} t_{-1,0}^{2}}{t_{0,0}^{1} t_{0,0}^{2}}
\end{array}\right), \\
B & =\left(\begin{array}{cccc}
1 & \frac{1}{\lambda} \frac{t_{0,1}^{1} t_{0,0}^{1}}{t_{0,1}^{0} t_{0,0}^{2}} & \frac{1}{\lambda} \frac{\left(t_{0,1}^{1}\right)^{2} t_{-1,0}^{1}}{t_{0,0}^{0} t_{0,1}^{0} t_{0,0}^{1}} & \frac{1}{\lambda} \frac{t_{0,1}^{1} t_{-1,0}^{2} t_{0,1}^{2}}{t_{0,1}^{0} t_{0,0}^{1} t_{0,0}^{2}} \\
\frac{t_{0,0}^{0} t_{0,1}^{2}}{t_{0,0}^{1} t_{0,1}^{1}} & 1 & 0 & 0 \\
0 & \frac{t_{0,0}^{0} t_{0,0}^{1}}{t_{-1,1}^{1} t_{0,0}^{2}} & \frac{t_{0,1}^{1} t_{-1,0}^{1}}{t_{0,0}^{1} t_{-1,1}^{1}} & \frac{t_{0,0}^{0} t_{-1,1}^{2} t_{0,1}^{2}}{t_{-1,1}^{1} t_{0,0}^{1} t_{0,0}^{2}} \\
0 & \frac{\left(t_{0,0}^{1}\right)^{2}}{t_{-1,1}^{2} t_{0,0}^{2}} & 0 & \frac{t_{-1,0}^{2} t_{0,1}^{2}}{t_{0,0}^{2} t_{-1,1}^{2}}
\end{array}\right) .
\end{aligned}
$$

Remark 5.4. In Example 5.2 we give a Lax pair realized in $2 \times 2$ matrices, while general formula (5.20) generates $3 \times 3$ matrices. The matter is that in the Lax pair obtained directly from (5.20) we made in this case some additional reduction.

\subsection{Lax pair for systems corresponding to the algebras $A_{N}$}

Instead of boundary condition (5.8) we can use also the degenerate boundary conditions of the form

$$
t^{N_{L}-1}=0, \quad t^{N_{L}}=1
$$

at the left end-point and

$$
t^{N_{R}+1}=0, \quad t^{N_{R}}=1
$$

at the right end-point. The degenerate boundary conditions imply that the corresponding eigenfunctions are zero: $g^{N_{L}-1}=0$ and $\psi^{N_{R}}=0$. 
In order to obtain the system corresponding to the Cartan matrix $A_{N-1}$ we cut off the Hirota chain by imposing degenerate boundary conditions (5.21) at the point $N_{L}=0$ and (5.22) at $N_{R}=N$. The resulting reduction is as follows

$$
\begin{aligned}
& t_{0,0}^{1} t_{1,1}^{1}-t_{1,0}^{1} t_{0,1}^{1}=t_{0,1}^{2}, \quad t_{0,0}^{j} t_{1,1}^{j}-t_{1,0}^{j} t_{0,1}^{j}=t_{1,0}^{j-1} t_{0,1}^{j+1}, \quad 2 \leq j \leq N-2, \\
& t_{0,0}^{N-1} t_{1,1}^{N-1}-t_{1,0}^{N-1} t_{0,1}^{N-1}=t_{1,0}^{N-2} .
\end{aligned}
$$

In this case our algorithm gives the Lax pair found years ago in [21]. In order to formulate it introduce the eigenvector $P=\left(\psi^{0}, \psi^{1}, \ldots, \psi^{N-1}\right)^{T}$ and $N \times N$ matrices

$$
U=\left(\begin{array}{ccccc}
\frac{t_{1,0}^{1}}{t_{0,0}^{1}} & -1 & \ldots & 0 & 0 \\
0 & \frac{t_{1,0}^{2} t_{0,0}^{1}}{t_{0,0}^{2} t_{1,0}^{1}} & \ldots & 0 & 0 \\
& & \ldots & & \\
0 & 0 & \ldots & \frac{t_{1,0}^{N-1} t_{0,0}^{N-2}}{t_{0,0}^{N-1} t_{1,0}^{N-2}} & -1 \\
0 & 0 & \ldots & 0 & \frac{t_{0,0}^{N-1}}{t_{1,0}^{N-1}}
\end{array}\right), \quad V=\left(\begin{array}{cccccc}
1 & 0 & 0 & \ldots & 0 & 0 \\
\frac{t_{0,1}^{2}}{t_{0,0}^{1} t_{0,1}^{1}} & 1 & 0 & \ldots & 0 & 0 \\
0 & \frac{t_{0,1}^{3} t_{0,0}^{1}}{t_{0,0}^{2} t_{0,1}^{2}} & 1 & \ldots & 0 & 0 \\
& & \ldots & & & \\
0 & 0 & \ldots & 0 & \frac{t_{0,0}^{N-2}}{t_{0,0}^{N-1} t_{0,1}^{N-1}} & 1
\end{array}\right) .
$$

It is easy to check that the compatibility condition of the equations

$$
P_{1,0}=U P, \quad P_{0,1}=V P .
$$

leads to the system (5.23).

\subsection{Lax pair for systems corresponding to the algebras $B_{N}$}

We impose boundary condition (5.21) for $N_{L}=0$ and (5.10) for $N_{R}=N$. The resulting reduction is as follows

$$
\begin{aligned}
& t_{0,0}^{1} t_{1,1}^{1}-t_{1,0}^{1} t_{0,1}^{1}=t_{0,1}^{2}, \quad t_{0,0}^{j} t_{1,1}^{j}-t_{1,0}^{j} t_{0,1}^{j}=t_{1,0}^{j-1} t_{0,1}^{j+1}, \quad 2 \leq j \leq N-1, \\
& t_{0,0}^{N} t_{1,1}^{N}-t_{1,0}^{N} t_{0,1}^{N}=\left(t_{1,0}^{N-1}\right)^{2} .
\end{aligned}
$$

System (5.24) can be rewritten in form of (1.3) by changing the variables $t^{j}=e^{-u^{j}}$. It corresponds to the algebra $B_{N}$. The system admits a Lax pair. Let us denote $P=\left(\psi^{0}, \psi^{1}, \ldots, \psi^{N-1}\right.$, $\left.g^{0}, g^{1}, \ldots, g^{N-1}\right)^{T}$ and introduce $2 N \times 2 N$ matrices

$$
A=\left(\begin{array}{cccccccc}
\frac{t_{1,0}^{1}}{t_{0,0}^{1}} & -1 & 0 & \ldots & 0 & 0 & \ldots & 0 \\
0 & \frac{t_{1,0}^{2} t_{0,0}^{1}}{t_{0,0}^{1} t_{1,0}^{1}}-1 & \ldots & 0 & 0 & \ldots & 0 \\
0 & 0 & \ldots & \frac{t_{1,0}^{N} t_{0,0}^{N-1}}{t_{0,0}^{N} t_{1,0}^{N-1}} & (-1)^{N} \frac{t_{-1,0}^{1}}{t_{0,0}^{1}} & (-1)^{N-1} \frac{t_{1,0}^{1} t_{-1,0}^{2}}{t_{0,0}^{1} t_{0,0}^{2}} & \ldots & -\frac{t_{1,0}^{N-1} t_{-1,0}^{N}}{t_{0,0}^{N-1} t_{0,0}^{N}} \\
0 & 0 & \ldots & 0 & \frac{t_{-1,0}^{1}}{t_{0,0}^{1}} & 0 & \ldots & 0 \\
0 & 0 & \ldots & 0 & -\frac{t_{-1,0}^{1}}{t_{0,0}^{1}} & \frac{t_{1,0}^{1} t_{-1,0}^{2}}{t_{0,0}^{1} t_{0,0}^{2}} & \ldots & 0 \\
0 & 0 & \ldots & 0 & (-1)^{N-1} \frac{t_{-1,0}^{1}}{t_{0,0}^{1}} & (-1)^{N} \frac{t_{1,0}^{1} t_{-1,0}^{2}}{t_{0,0}^{1} t_{0,0}^{2}} & \ldots & \frac{t_{1,0}^{N-1} t_{-1,0}^{N}}{t_{0,0}^{N-1} t_{0,0}^{N}}
\end{array}\right),
$$




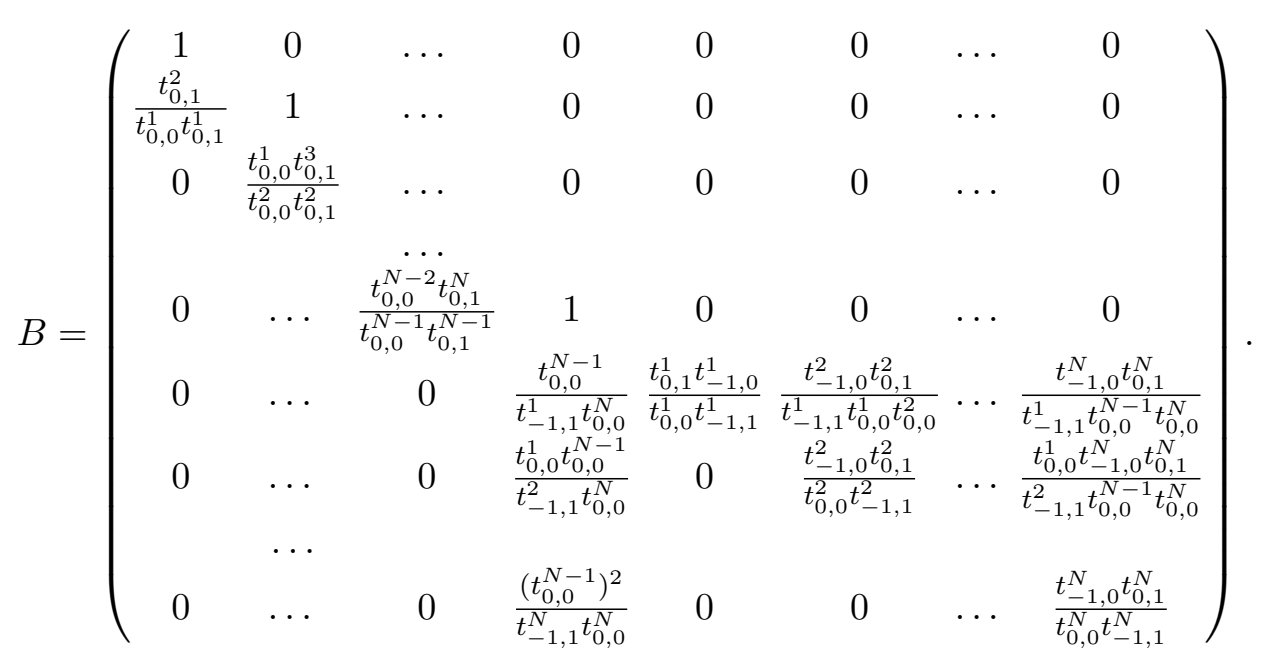

Then according to our general scheme the compatibility condition of the equations

$$
P_{1,0}=A P, \quad P_{0,1}=B P
$$

leads to the system (5.24).

Remark 5.5. The system $B_{N}$ can be obtained from the system $A_{2 N-1}$ by imposing the cutting off constraint of the form

$$
t_{0,1}^{N+1}=t_{1,0}^{N-1}
$$

(see Lemma 5.1 above).

\section{Method of finding integrals from Lax representation for the systems corresponding to the Cartan matrices $A_{N}, B_{N}$}

In this section we show that the Lax pair allows one to generate integrals for the systems corresponding to the simple Lie algebras $A_{N}, B_{N}$. Concentrate on $m$-integrals. Due to the definition we have two different expression for the shifted eigenvector $P_{k, 1}, k \geq 1$

$$
P_{k, 1}=V_{k, 0} U_{k-1,0} U_{k-2,0} \cdots U_{1,0} U_{0,0} P,
$$

and similarly

$$
P_{k, 1}=U_{k-1,1} U_{k-2,1} \cdots U_{1,1} U_{0,1} V_{0,0} P .
$$

Comparison of these two formulas yields

$$
D_{m}\left(U_{k-1,0} U_{k-2,0} \cdots U_{1,0} U_{0,0}\right)=V_{k, 0} U_{k-1,0} U_{k-2,0} \cdots U_{1,0} U_{0,0} V_{0,0}^{-1} .
$$

Due to the triangular structure of the matrices $V_{k, 0}$ and $V_{0,0}$ the map converting any upper triangular matrix $X$ to a matrix $\bar{X}=V_{k, 0} X V_{0,0}^{-1}$ leaves unchanged the element of the matrix $X$ located at the right upper corner: $(X)_{1, N}=\bar{X}_{1, N}$. Thus the corresponding element, denote it through $I_{(k-N)}(k>N)$, of the upper triangular matrix $U_{k-1,0} U_{k-2,0} \cdots U_{1,0} U_{0,0}$ is an $m$ integral. In such a way we get a set of integrals $I_{(1)}, I_{(2)}, \ldots, I_{(N)}$. Examples below show that they constitute a complete set of $m$-integrals, however we are not able to prove this fact in general. In a similar way one can find integrals in the other direction.

Let us illustrate the statement above with the following 
Example 6.1. Consider the system (5.23) for $N=3$

$$
t_{0,0}^{1} t_{1,1}^{1}-t_{1,0}^{1} t_{0,1}^{1}=t_{0,1}^{2}, \quad t_{0,0}^{2} t_{1,1}^{2}-t_{1,0}^{2} t_{0,1}^{2}=t_{1,0}^{1} .
$$

Recall its Lax pair

$$
P_{1,0}=U P, \quad P_{0,1}=V P,
$$

where $P=\left(\psi^{0}, \psi^{1}, \psi^{2}\right)^{T}$ and

$$
U=\left(\begin{array}{ccc}
\frac{t_{1,0}^{1}}{t_{0,0}^{1}} & -1 & 0 \\
0 & \frac{t_{1,0}^{2} t_{0,0}^{1}}{t_{0,0}^{2} t_{1,0}^{1}} & -1 \\
0 & 0 & \frac{t_{0,0}^{2}}{t_{1,0}^{2}}
\end{array}\right), \quad V=\left(\begin{array}{ccc}
1 & 0 & 0 \\
\frac{t_{0,1}^{2}}{t_{0,0}^{1} t_{0,1}^{1}} & 1 & 0 \\
0 & \frac{t_{0,0}^{1}}{t_{0,0}^{2} t_{0,1}^{2}} & 1
\end{array}\right) .
$$

Evaluate the elements at the right upper corner for the following two products $U_{2,0} U_{1,0} U_{0,0}$ and $U_{3,0} U_{2,0} U_{1,0} U_{0,0}$ and find two independent $m$-integrals

$$
I_{(1)}=\frac{t_{3}^{1}}{t_{2}^{1}}+\frac{t_{1}^{1} t_{2}^{2}}{t_{2}^{1} t_{1}^{2}}+\frac{t_{0}^{2}}{t_{1}^{2}}, \quad I_{(2)}=\frac{t_{4}^{1}}{t_{3}^{1}} I_{(1)}+\frac{t_{1}^{1} t_{3}^{2}}{t_{3}^{1} t_{1}^{2}}+\frac{t_{2}^{1} t_{0}^{2} t_{3}^{2}}{t_{3}^{1} t_{1}^{2} t_{2}^{2}}+\frac{t_{0}^{2}}{t_{2}^{2}},
$$

where the second index for the variables $t^{1}, t^{2}$ is omitted. Since the integral $I_{(2)}$ is too complicated one can choose a more simple one

$$
\tilde{I}_{(2)}=D_{n}^{-1}\left(I_{(1)} D_{n} I_{(1)}-I_{(2)}\right)=\frac{t_{0}^{1}}{t_{1}^{1}}+\frac{t_{2}^{1} t_{0}^{2}}{t_{1}^{1} t_{1}^{2}}+\frac{t_{2}^{2}}{t_{1}^{2}} .
$$

By using Theorem 1.2 one can check that integrals $I_{(1)}$ and $\widetilde{I}_{(2)}$ provide a complete set of integrals.

In a similar way integrals for the system (5.24), corresponding to the algebra $B_{N}$, are constructed. Let us consider a matrix $\Phi$ of dimension $2 N \times 2 N$

$$
\Phi=\left(\begin{array}{ll}
E_{11} & E_{12} \\
E_{21} & E_{22}
\end{array}\right)
$$

where $E_{11}$ is the unity matrix of dimension $N, E_{12}, E_{21}$ are matrices of dimension $N$ with zero entries, $E_{22}$ is a matrix of dimension $N$ with the unity adverse diagonal. By an automorphism $X \rightarrow \Phi X \Phi^{-1}$ the matrices (5.25) and (5.26) are transformed to the triangular matrices

$$
\bar{A}=\left(\begin{array}{cccccccc}
\frac{t_{1,0}^{1}}{t_{0,0}^{1}} & -1 & 0 & \ldots & 0 & 0 & \ldots & 0 \\
0 & \frac{t_{1,0}^{2} t_{0,0}^{1}}{t_{0,0}^{2} t_{1,0}^{1}}-1 & \ldots & 0 & 0 & \ldots & 0 \\
0 & 0 & \ldots & \frac{t_{1,0}^{N} t_{0,0}^{N-1}}{t_{0,0}^{N} t_{1,0}^{N-1}} & -\frac{t_{1,0}^{N-1} t_{-1,0}^{N}}{t_{0,0}^{N-1} t_{0,0}^{N}} & \frac{t_{1,0}^{N-2} t_{-1,0}^{N-1}}{t_{0,0}^{N-2} t_{0,0}^{N-1}} & \ldots & (-1)^{N} \frac{t_{-1,0}^{1}}{t_{0,0}^{1}} \\
0 & 0 & \ldots & 0 & \frac{t_{1,0}^{N-1} t_{-1,0}^{N}}{t_{0,0}^{N-1} t_{0,0}^{N}} & -\frac{t_{1,0}^{N-2} t_{-1,0}^{N-1}}{t_{0,0}^{N-2} t_{0,0}^{N-1}} & \ldots & (-1)^{N-1} \frac{t_{-1,0}^{1}}{t_{0,0}^{1}} \\
0 & 0 & \ldots & 0 & 0 & \frac{t_{1,0}^{N-2} t_{-1,0}^{N-1}}{t_{0,0}^{N-2} t_{0,0}^{N-1}} & \ldots & (-1)^{N} \frac{t_{-1,0}^{1}}{t_{0,0}^{1}} \\
0 & 0 & \ldots & 0 & 0 & 0 & \ldots & \frac{t_{-1,0}^{1}}{t_{0,0}^{1}}
\end{array}\right),
$$




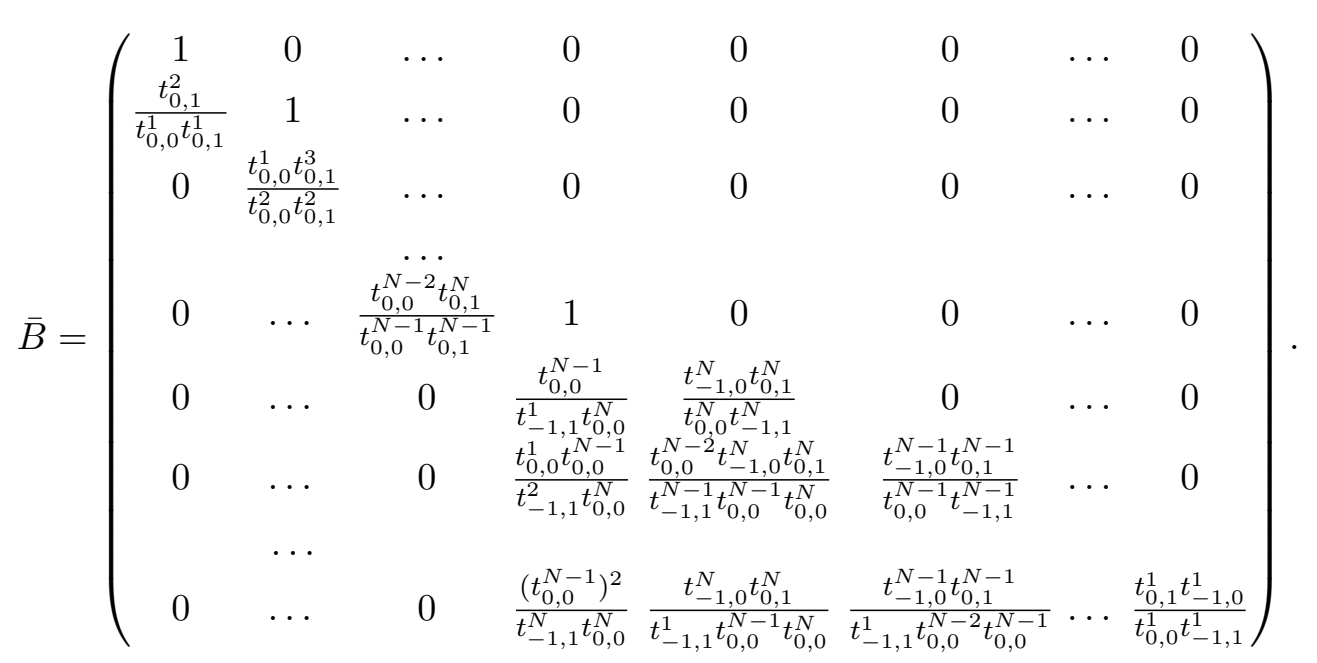

By an automorphism acting as follows $X \rightarrow F^{-1} X F$ matrix (6.2) is transformed to a lower triangular matrix for which all diagonal entries are equal to the unity. Here $F$ is a $2 N \times 2 N$ matrix of the form

$$
F=\left(\begin{array}{ll}
E_{11} & E_{12} \\
E_{21} & F_{22}
\end{array}\right)
$$

$F_{22}$ is a diagonal matrix such that $F_{22}=\operatorname{diag}\left(\frac{t_{0,0}^{N}}{t_{-1,0}^{N}}, \frac{t_{0,0}^{N-1}}{t_{-1,0}^{N-1}}, \ldots, \frac{t_{0,0}^{1}}{t_{-1,0}^{1}}\right)$ and $E_{i, j}$ are defined in (6.1). Reasonings similar to that of the case $A_{N}$ allow one to derive the integrals.

Example 6.2. Consider the system (5.24) for $N=2$

$$
t_{0,0}^{1} t_{1,1}^{1}-t_{1,0}^{1} t_{0,1}^{1}=t_{0,1}^{2}, \quad t_{0,0}^{2} t_{1,1}^{2}-t_{1,0}^{2} t_{0,1}^{2}=\left(t_{1,0}^{1}\right)^{2} .
$$

Recall its Lax pair

$$
P_{1,0}=A P, \quad P_{0,1}=B P,
$$

where $P=\left(\psi^{0}, \psi^{1}, g^{0}, g^{1}\right)^{T}$ and

$$
A=\left(\begin{array}{cccc}
\frac{t_{1,0}^{1}}{t_{0,0}^{1}} & -1 & 0 & 0 \\
0 & \frac{t_{1,0}^{2} t_{0,0}^{1}}{t_{0,0}^{2} t_{1,0}^{1}} & \frac{t_{-1,0}^{1}}{t_{0,0}^{1}} & -\frac{t_{1,0}^{1} t_{-1,0}^{2}}{t_{0,0}^{1} t_{0,0}^{2}} \\
0 & 0 & \frac{t_{-1,0}^{1}}{t_{0,0}^{1}} & 0 \\
0 & 0 & -\frac{t_{-1,0}^{1}}{t_{0,0}^{1}} & \frac{t_{1,0}^{1} t_{-1,0}^{2}}{t_{0,0}^{1} t_{0,0}^{2}}
\end{array}\right), \quad B=\left(\begin{array}{cccc}
1 & 0 & 0 & 0 \\
\frac{t_{0,1}^{2}}{t_{0,0}^{1} t_{0,1}^{1}} & 1 & 0 & 0 \\
0 & \frac{t_{0,0}^{1}}{t_{-1,1}^{1} t_{0,0}^{2}} & \frac{t_{0,1}^{1} t_{-1,0}^{1}}{t_{0,0}^{1} t_{-1,1}^{1}} & \frac{t_{-1,0}^{2} t_{0,1}^{2}}{t_{-1,1}^{1} t_{0,0}^{1} t_{0,0}^{2}} \\
0 & \frac{\left(t_{0,0}^{1}\right)^{2}}{t_{-1,1}^{2} t_{0,0}^{2}} & 0 & \frac{t_{-1,0}^{2} t_{0,1}^{2}}{t_{0,0}^{2} t_{-1,1}^{2}}
\end{array}\right) .
$$

By an automorphism $X \rightarrow \Phi X \Phi^{-1}$ transform the matrices $A$ and $B$ to the triangular matrices

$$
\bar{A}=\left(\begin{array}{cccc}
\frac{t_{1,0}^{1}}{t_{0,0}^{1}} & -1 & 0 & 0 \\
0 & \frac{t_{1,0}^{2} t_{0,0}^{1}}{t_{0,0}^{2} t_{1,0}^{1}} & -\frac{t_{1,0}^{1} t_{-1,0}^{2}}{t_{0,0}^{1} t_{0,0}^{2}} & \frac{t_{-1,0}^{1}}{t_{0,0}^{1}} \\
0 & 0 & \frac{t_{1,0}^{1} t_{-1,0}^{2}}{t_{0,0}^{1} t_{0,0}^{2}} & -\frac{t_{-1,0}^{1}}{t_{0,0}^{1}} \\
0 & 0 & 0 & \frac{t_{-1,0}^{1}}{t_{0,0}^{1}}
\end{array}\right), \quad \bar{B}=\left(\begin{array}{cccc}
1 & 0 & 0 & 0 \\
\frac{t_{0,1}^{2}}{t_{0,0}^{1} t_{0,1}^{1}} & 1 & 0 & 0 \\
0 & \frac{\left(t_{0,0}^{1}\right)^{2}}{t_{-1,1}^{2} t_{0,0}^{2}} & \frac{t_{-1,0}^{2} t_{0,1}^{2}}{t_{0,0}^{2} t_{-1,1}^{2}} & 0 \\
0 & \frac{t_{0,0}^{1}}{t_{-1,1}^{1} t_{0,0}^{2}} \frac{t_{-1,0}^{2} t_{0,1}^{2}}{t_{-1,1}^{1} t_{0,0}^{1} t_{0,0}^{2}} \frac{t_{-1,0}^{1} t_{0,1}^{1}}{t_{-1,1}^{1} t_{0,0}^{1}}
\end{array}\right) .
$$


By automorphism $X \rightarrow F^{-1} X F$ transform the matrices (6.3) to the triangular matrices

$$
\hat{A}=\left(\begin{array}{cccc}
\frac{t_{1,0}^{1}}{t_{0,0}^{1}} & -1 & 0 & 0 \\
0 & \frac{t_{1,0}^{2} t_{0,0}^{1}}{t_{0,0}^{2} t_{1,0}^{1}} & -\frac{t_{1,0}^{1}}{t_{0,0}^{1}} & 1 \\
0 & 0 & \frac{t_{1,0}^{1} t_{0,0}^{2}}{t_{0,0}^{1} t_{1,0}^{2}} & -\frac{t_{0,0}^{2}}{t_{1,0}^{2}} \\
0 & 0 & 0 & \frac{t_{0,0}^{1}}{t_{1,0}^{1}}
\end{array}\right), \quad \hat{B}=\left(\begin{array}{cccc}
1 & 0 & 0 & 0 \\
\frac{t_{0,1}^{2}}{t_{0,0}^{1} t_{0,1}^{1}} & 1 & 0 & 0 \\
0 & \frac{\left(t_{0,0}^{1}\right)^{2}}{t_{0,0}^{2} t_{0,1}^{2}} & 1 & 0 \\
0 & \frac{t_{0,0}^{1}}{t_{0,1}^{1} t_{0,0}^{2}} & \frac{t_{0,1}^{2}}{t_{0,0}^{1} t_{0,1}^{1}} & 1
\end{array}\right) .
$$

Evaluate the elements at the right upper corner for the following products $\hat{A}_{2,0} \hat{A}_{1,0} \hat{A}_{0,0}$ and $\hat{A}_{3,0} \hat{A}_{2,0} \hat{A}_{1,0} \hat{A}_{0,0}$ and find two independent $m$-integrals

$$
\begin{aligned}
& I_{(1)}=-\frac{t_{3}^{1}}{t_{2}^{1}}-\frac{t_{2}^{1} t_{0}^{2}}{t_{1}^{1} t_{1}^{2}}-\frac{t_{1}^{1} t_{2}^{2}}{t_{2}^{1} t_{1}^{2}}-\frac{t_{0}^{1}}{t_{1}^{1}}, \\
& I_{(2)}=-\frac{t_{4}^{1}}{t_{2}^{1}}-\frac{t_{1}^{1} t_{2}^{2} t_{4}^{1}}{t_{2}^{1} t_{1}^{2} t_{3}^{1}}-\frac{t_{1}^{1} t_{3}^{2}}{t_{3}^{1} t_{1}^{2}}-\frac{t_{2}^{1} t_{0}^{2} t_{4}^{1}}{t_{1}^{1} t_{1}^{2} t_{3}^{1}}-\frac{\left(t_{2}^{1}\right)^{2} t_{0}^{2} t_{3}^{2}}{t_{1}^{1} t_{1}^{2} t_{3}^{1} t_{2}^{2}}-\frac{t_{3}^{1} t_{0}^{2}}{t_{1}^{1} t_{2}^{2}}-\frac{t_{0}^{1} t_{4}^{1}}{t_{1}^{1} t_{3}^{1}}-\frac{t_{0}^{1} t_{2}^{1} t_{3}^{2}}{t_{1}^{1} t_{2}^{2} t_{3}^{1}}-\frac{t_{0}^{1} t_{3}^{1} t_{1}^{2}}{t_{1}^{1} t_{2}^{2} t_{2}^{1}}-\frac{t_{0}^{1}}{t_{2}^{1}} .
\end{aligned}
$$

Replace the integral $I_{(2)}$ by a more simple one

$$
\tilde{I}_{(2)}=I_{(2)}+I_{(1)} D_{n} I_{(1)}=\frac{t_{0}^{2}}{t_{1}^{2}}+\frac{\left(t_{1}^{1}\right)^{2} t_{2}^{2}}{\left(t_{2}^{1}\right)^{2} t_{1}^{2}}+2 \frac{t_{3}^{1} t_{1}^{1}}{\left(t_{2}^{1}\right)^{2}}+\frac{\left(t_{3}^{1}\right)^{2} t_{1}^{2}}{\left(t_{2}^{1}\right)^{2} t_{2}^{2}}+\frac{t_{3}^{2}}{t_{2}^{2}} .
$$

Here the second index for the variables $t^{1}, t^{2}$ is omitted, since its values is zero for all considered variables. It can be proved by using Theorem 1.2 that integrals $I_{(1)}$ and $\widetilde{I}_{(2)}$ constitute a complete set of integrals.

\section{$7 \quad$ Periodic boundary conditions}

In this section we discuss briefly the well known periodically closed reduction of the Hirota chain (see for more details and the references [41]). Close the chain (5.1) by imposing the periodical boundary conditions

$$
t^{-1}=t^{N}, \quad t^{N+1}=t^{0} .
$$

Close the Lax pair (5.2) by setting the conditions on the eigenfunctions

$$
\psi^{-1}=\lambda \psi^{N}, \quad \psi^{N+1}=\frac{1}{\lambda} \psi^{0} .
$$

As a result we get a finite system of the form

$$
\begin{aligned}
& t_{0,0}^{0} t_{1,1}^{0}-t_{1,0}^{0} t_{0,1}^{0}=t_{1,0}^{N} t_{0,1}^{1}, \quad t_{0,0}^{j} t_{1,1}^{j}-t_{1,0}^{j} t_{0,1}^{j}=t_{1,0}^{j-1} t_{0,1}^{j+1}, \quad 1 \leq j \leq N-1, \\
& t_{0,0}^{N} t_{1,1}^{N}-t_{1,0}^{N} t_{0,1}^{N}=t_{1,0}^{N-1} t_{0,1}^{0},
\end{aligned}
$$

which is closely connected with the Cartan matrix $A_{N}^{(1)}$.

Boundary conditions (7.1) reduce the sequence of linear discrete equations (5.2) to a Lax pair for the reduced system (7.2). Introduce the eigenvector $P=\left(\psi^{0}, \psi^{1}, \ldots, \psi^{N}\right)^{T}$ and $N+1 \times N+1$ matrices

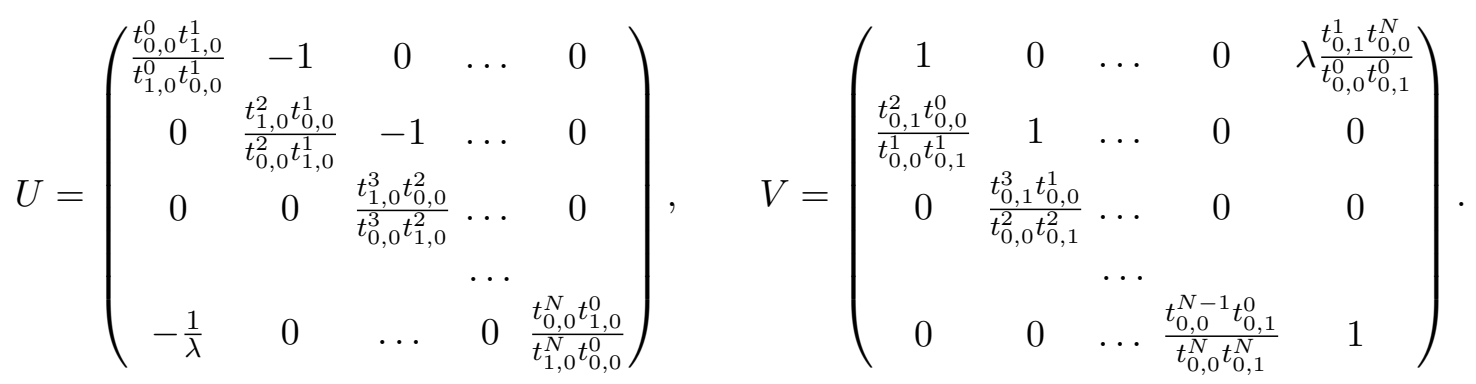


If the functions $t^{0}, t^{1}, \ldots, t^{N}$ solve the system (7.2) then the overdetermined system of linear equations

$$
P_{1,0}=U P, \quad P_{0,1}=V P
$$

is compatible (see [41]).

Remark 7.1. Note that system (7.2) differs from the system corresponding to the same algebra $A_{N}^{(1)}$ but defined by the formula (1.3).

For example the system (7.2) for $N=2$ is of the form

$$
\begin{aligned}
& t_{0,0}^{0} t_{1,1}^{0}-t_{1,0}^{0} t_{0,1}^{0}=t_{1,0}^{2} t_{0,1}^{1}, \quad t_{0,0}^{1} t_{1,1}^{1}-t_{1,0}^{1} t_{0,1}^{1}=t_{1,0}^{0} t_{0,1}^{2}, \\
& t_{0,0}^{2} t_{1,1}^{2}-t_{1,0}^{2} t_{0,1}^{2}=t_{1,0}^{1} t_{0,1}^{0} .
\end{aligned}
$$

In terms of the variables $u^{0}=-\log t^{0}, u^{1}=-\log t^{1}, u^{2}=-\log t^{2}$ it looks like

$$
\begin{aligned}
& \Delta\left(u^{0}\right)=e^{u_{1,0}^{0}+u_{0,1}^{0}-u_{0,1}^{1}-u_{1,0}^{2}}, \quad \Delta\left(u^{1}\right)=e^{-u_{1,0}^{0}+u_{1,0}^{1}+u_{0,1}^{1}-u_{0,1}^{2},} \\
& \Delta\left(u^{2}\right)=e^{-u_{0,1}^{0}-u_{1,0}^{1}+u_{1,0}^{2}+u_{0,1}^{2}},
\end{aligned}
$$

while formula (1.3) gives the system

$$
\begin{aligned}
& \Delta\left(u^{0}\right)=e^{u_{1,0}^{0}+u_{0,1}^{0}-u_{1,0}^{1}-u_{1,0}^{2}}, \quad \Delta\left(u^{1}\right)=e^{-u_{0,1}^{0}+u_{1,0}^{1}+u_{0,1}^{1}-u_{1,0}^{2},} \\
& \Delta\left(u^{2}\right)=e^{-u_{0,1}^{0}-u_{0,1}^{1}+u_{1,0}^{2}+u_{0,1}^{2}} .
\end{aligned}
$$

After permutations $n \leftrightarrow m$ we get

$$
\begin{aligned}
& \Delta\left(u^{0}\right)=e^{u_{1,0}^{0}+u_{0,1}^{0}-u_{0,1}^{1}-u_{0,1}^{2}}, \quad \Delta\left(u^{1}\right)=e^{-u_{1,0}^{0}+u_{1,0}^{1}+u_{0,1}^{1}-u_{0,1}^{2},} \\
& \Delta\left(u^{2}\right)=e^{-u_{1,0}^{0}-u_{1,0}^{1}+u_{1,0}^{2}+u_{0,1}^{2}} .
\end{aligned}
$$

Obviously, systems (7.5) and (7.6) are different.

Let us give also the Lax pair for the system (7.4). Let us consider vector $P=\left(\psi^{0}, \psi^{1}, \psi^{2}\right)^{T}$. Introduce $3 \times 3$ matrices

$$
U=\left(\begin{array}{ccc}
\frac{t_{0,0}^{0} t_{1,0}^{1}}{t_{1,0}^{0} t_{0,0}^{1}} & -1 & 0 \\
0 & \frac{t_{1,0}^{2} t_{0,0}^{1}}{t_{0,0}^{2} t_{1,0}^{1}} & -1 \\
-\frac{1}{\lambda} & 0 & \frac{t_{0,0}^{2} t_{1,0}^{0}}{t_{1,0}^{2} t_{0,0}^{0}}
\end{array}\right), \quad V=\left(\begin{array}{ccc}
1 & 0 & \lambda \frac{t_{0,1}^{1} t_{0,0}^{2}}{t_{0,0}^{0} t_{0,1}^{0}} \\
\frac{t_{0,1}^{2} t_{0,0}^{0}}{t_{0,0}^{1} t_{0,1}^{1}} & 1 & 0 \\
0 & \frac{t_{0,1}^{0} t_{0,0}^{1}}{t_{0,0}^{2} t_{0,1}^{2}} & 1
\end{array}\right) .
$$

If the functions $t^{0}, t^{1}, t^{2}$ satisfy the system (7.4) then the equations

$$
P_{1,0}=U P, \quad P_{0,1}=V P .
$$

are compatible.

Remark 7.2. The Lax pair (7.3) can be rewritten in terms of the Cartan-Weyl basis of the algebra $A_{N}^{(1)}$

$$
P_{1,0}=\left(-\Lambda+e^{U_{1,0}-U_{0,0}}\right) P, \quad P_{0,1}=\left(E+e^{U_{0,1}} \bar{\Lambda} e^{-U_{0,0}}\right) P,
$$

where $\Lambda=\sum_{i=0}^{N} f_{i}, \bar{\Lambda}=\sum_{i=0}^{N} e_{i}, U=\sum_{i=0}^{N} u^{i} h_{i}, e^{-u^{i}}=t^{i}$,

$$
\left[h_{i}, h_{j}\right]=0, \quad\left[e_{i}, f_{j}\right]=\delta_{i j} h_{i}, \quad\left[h_{i}, e_{j}\right]=A_{i j} e_{j}, \quad\left[h_{i}, f_{j}\right]=-A_{i j} f_{j},
$$

and $A_{i j}$ are elements of the Cartan matrix of the algebra $A_{N}^{(1)}$. 


\section{Conclusions}

A map is suggested converting any $N \times N$ matrix $A$ to a finite system of difference-difference equations of exponential type (see (1.3)). A hypothesis is formulated claiming that if $A$ coincides with the Cartan matrix of a finite or affine Lie algebra then the corresponding system is integrable. The hypothesis is approved by numerous examples. The systems obtained are rather simple and elegant. They essentially differ from those studied earlier (see survey [25] and references therein). For instance, the system corresponding the algebra $G_{2}$ given in [25] reads as

$$
\begin{aligned}
& T_{m}^{(1)}(u-1) T_{m}^{(1)}(u+1)=T_{m-1}^{(1)}(u) T_{m+1}^{(1)}(u)+T_{3 m}^{(2)}(u), \\
& T_{3 m}^{(2)}\left(u-\frac{1}{3}\right) T_{3 m}^{(2)}\left(u+\frac{1}{3}\right)=T_{3 m-1}^{(2)}(u) T_{3 m+1}^{(2)}(u)+T_{m}^{(1)}\left(u-\frac{2}{3}\right) T_{m}^{(1)}(u) T_{m}^{(1)}\left(u+\frac{2}{3}\right), \\
& T_{3 m+1}^{(2)}\left(u-\frac{1}{3}\right) T_{3 m+1}^{(2)}\left(u+\frac{1}{3}\right) \\
& \quad=T_{3 m}^{(2)}(u) T_{3 m+2}^{(2)}(u)+T_{m}^{(1)}\left(u-\frac{1}{3}\right) T_{m}^{(1)}\left(u+\frac{1}{3}\right) T_{m+1}^{(1)}(u), \\
& T_{3 m+2}^{(2)}\left(u-\frac{1}{3}\right) T_{3 m+2}^{(2)}\left(u+\frac{1}{3}\right) \\
& \quad=T_{3 m+1}^{(2)}(u) T_{3 m+3}^{(2)}(u)+T_{m}^{(1)}(u) T_{m+1}^{(1)}\left(u-\frac{1}{3}\right) T_{m+1}^{(1)}\left(u+\frac{1}{3}\right),
\end{aligned}
$$

while our formula (1.3) generates $G_{2}$ system which can be represented as follows

$$
t_{n, m}^{1} t_{n+1, m+1}^{1}-t_{n+1, m}^{1} t_{n, m+1}^{1}=t_{n, m+1}^{2}, \quad t_{n, m}^{2} t_{n+1, m+1}^{2}-t_{n+1, m}^{2} t_{n, m+1}^{2}=\left(t_{n+1, m}^{1}\right)^{3} .
$$

In a recent article by Kimura, Yamashita and Nakamura [23] a new application of conserved quantities of discrete-time integrable systems to numerical computations is suggested. The systems studied in the present paper might have applications in such kind numerical methods.

\section{Acknowledgments}

The authors are grateful to the referees for their important contribution to improve the article. This work is partially supported by Russian Foundation for Basic Research (RFBR) grants 11-01-97005-r-povoljie-a, 12-01-31208-mol_a and 10-01-00088-a and by Federal Task Program "Scientific and pedagogical staff of innovative Russia for 2009-2013" contract no. 2012-1.5-12000-1003-011.

\section{References}

[1] Adler V.E., Bobenko A.I., Suris Y.B., Classification of integrable equations on quad-graphs. The consistency approach, Comm. Math. Phys. 233 (2003), 513-543, nlin.SI/0202024.

[2] Adler V.E., Startsev S.Y., On discrete analogues of the Liouville equation, Theoret. and Math. Phys. 121 (1999), 1484-1495, solv-int/9902016.

[3] Belavin A.A., Polyakov A.M., Zamolodchikov A.B., Infinite conformal symmetry in two-dimensional quantum field theory, Nuclear Phys. B 241 (1984), 333-380.

[4] Bobenko A.I., Suris Y.B., Integrable systems on quad-graphs, Int. Math. Res. Not. (2002), 573-611, nlin.SI/0110004.

[5] Bogoyavlensky O.I., On perturbations of the periodic Toda lattice, Comm. Math. Phys. 51 (1976), $201-209$.

[6] Corrigan E., Recent developments in affine Toda quantum field theory, in Particles and Fields (Banff, AB, 1994), CRM Ser. Math. Phys., Springer, New York, 1999, 1-34, hep-th/9412213. 
[7] Date E., Jimbo M., Miwa T., Method for generating discrete soliton equations. II, J. Phys. Soc. Japan 52 (1983), 4125-4131.

[8] Doliwa A., Geometric discretisation of the Toda system, Phys. Lett. A 234 (1997), 187-192, solv-int/9612006.

[9] Drinfel'd V.G., Sokolov V.V., Lie algebras and equations of Korteweg-de Vries type, J. Math. Sci. 30 (1985), 1975-2036.

[10] Fordy A.P., Gibbons J., Integrable nonlinear Klein-Gordon equations and Toda lattices, Comm. Math. Phys. 77 (1980), 21-30.

[11] Ganzha E.I., Tsarev S.P., Integration of classical series $A_{n}, B_{n}, C_{n}$, of exponential systems, Krasnoyarsk State Pedagogical University, Krasnoyarsk, 2001.

[12] Garifullin R.N., Gudkova E.V., Habibullin I.T., Method for searching higher symmetries for quad-graph equations, J. Phys. A: Math. Theor. 44 (2011), 325202, 16 pages, arXiv:1104.0493.

[13] Guryeva A.M., Zhiber A.V., On the characteristic equations of a system of quasilinear hyperbolic equations, Vestnik UGATU 6 (2005), 26-34.

[14] Habibullin I.T., Characteristic algebras of fully discrete hyperbolic type equations, SIGMA 1 (2005), 023, 9 pages, nlin.SI/0506027.

[15] Habibullin I.T., Discrete chains of the series C, Theoret. and Math. Phys. 146 (2006), 170-182.

[16] Habibullin I.T., Gudkova E.V., Boundary conditions for multidimensional integrable equations, Funct. Anal. Appl. 38 (2004), 138-148.

[17] Habibullin I.T., Pekan A., Characteristic Lie algebra and the classification of semi-discrete models, Theoret. and Math. Phys. 151 (2007), 781-790, nlin.SI/0610074.

[18] Habibullin I.T., Zheltukhin K., Yangubaeva M., Cartan matrices and integrable lattice Toda field equations, J. Phys. A: Math. Theor. 44 (2011), 465202, 20 pages, arXiv:1105.4446.

[19] Habibullin I.T., Zheltukhina N., Sakieva A., Discretization of hyperbolic type Darboux integrable equations preserving integrability, J. Math. Phys. 52 (2011), 093507, 12 pages, arXiv:1102.1236.

[20] Habibullin I.T., Zheltukhina N., Sakieva A., On Darboux-integrable semi-discrete chains, J. Phys. A: Math. Theor. 43 (2010), 434017, 14 pages, arXiv:0907.3785.

[21] Hirota R., Discrete two-dimensional Toda molecule equation, J. Phys. Soc. Japan 56 (1987), 4285-4288.

[22] Inoue R., Hikami K., The lattice Toda field theory for simple Lie algebras: Hamiltonian structure and $\tau$-function, Nuclear Phys. B $\mathbf{5 8 1}$ (2000), 761-775.

[23] Kimura K., Yamashita T., Nakamura Y., Conserved quantities of the discrete finite Toda equation and lower bounds of the minimal singular value of upper bidiagonal matrices, J. Phys. A: Math. Theor. 44 (2011), 285207, 12 pages.

[24] Kuniba A., Nakanishi T., Suzuki J., Functional relations in solvable lattice models. I. Functional relations and representation theory, Internat. J. Modern Phys. A 9 (1994), 5215-5266, hep-th/9309137.

[25] Kuniba A., Nakanishi T., Suzuki J., T-systems and $Y$-systems in integrable systems, J. Phys. A: Math. Theor. 44 (2011), 103001, 146 pages, arXiv:1010.1344.

[26] Levi D., Yamilov R.I., Generalized symmetry integrability test for discrete equations on the square lattice, J. Phys. A: Math. Theor. 44 (2011), 145207, 22 pages, arXiv:1011.0070.

[27] Leznov A.N., Savel'ev M.V., Group methods for the integration of nonlinear dynamical systems, Nauka, Moscow, 1985.

[28] Leznov A.N., Smirnov V.G., Shabat A.B., Internal symmetry group and integrability conditions for twodimensional dynamical systems, Theoret. and Math. Phys. 51 (1982), 322-330.

[29] Mikhailov A.V., Integrability of a two-dimensional generalization of the Toda chain, JETP Lett. 30 (1979), 414-418.

[30] Mikhailov A.V., Olshanetsky M.A., Perelomov A.M., Two-dimensional generalized Toda lattice, Comm. Math. Phys. 79 (1981), 473-488.

[31] Nieszporski M., A Laplace ladder diagram of discrete Laplace-type equations, Theoret. and Math. Phys. 133 (2002), 1576-1584.

[32] Nijhoff F.W., Lax pair for the Adler (lattice Krichever-Novikov) system, Phys. Lett. A 297 (2002), 49-58, nlin.SI/0110027. 
[33] Novikov S.P., Dynnikov I.A., Discrete spectral symmetries of small-dimensional differential operators and difference operators on regular lattices and two-dimensional manifolds, Russian Math. Surveys 52 (1997), 1057-1116, math-ph/0003009.

[34] Olshanetsky M.A., Perelomov A.M., Classical integrable finite-dimensional systems related to Lie algebras, Phys. Rep. 71 (1981), 313-400.

[35] Shabat A.B., Higher symmetries of two-dimensional lattices, Phys. Lett. A 200 (1995), 121-133.

[36] Shabat A.B., Yamilov R.I., Exponential systems of type I and the Cartan matrices, Preprint, Bashkirian Branch of Academy of Science of the USSR, Ufa, 1981.

[37] Smirnov S.V., Semidiscrete Toda lattices, arXiv:1203.1764.

[38] Suris Y.B., Generalized Toda chains in discrete time, Leningrad Math. J. 2 (1991), 339-352.

[39] Tsuboi Z., Solutions of discretized affine Toda field equations for $A_{n}^{(1)}, B_{n}^{(1)}, C_{n}^{(1)}, D_{n}^{(1)}, A_{n}^{(2)}$ and $D_{n+1}^{(2)}$, J. Phys. Soc. Japan 66 (1997), 3391-3398, solv-int/9610011.

[40] Ward R.S., Discrete Toda field equations, Phys. Lett. A 199 (1995), 45-48, solv-int/9502002.

[41] Zabrodin A.V., Hirota's difference equations, Theoret. and Math. Phys. 113 (1997), 1347-1392, solv-int/9704001. 